\title{
Connections between asteroids and cometary nuclei
}

\author{
Imre Toth \\ Konkoly Observatory, Budapest, P.O. Box 67, H-1525, Hungary \\ email: tothi@konkoly.hu
}

\begin{abstract}
We review the recent progress in the exploration of the interrelations between primitive small bodies of the solar system which are preserved the pristine material in their interior: cometary nuclei, Transneptunian Objects, Centaurs, and primitive asteroids, and they are considered as primordial objects. In addition, we discuss the properties of the asteroid-comet transition objects which have really enigmatic behavior. The comets have most primitive, accessible material in the solar system but we do not know what is hidden below the evolved surface layers. Comets must become dormant but we do not know whether the ice is exhausted or sublimation is inhibited (blocked by quenching mechanisms). There must be many dormant comets masquerading as asteroids but we do not know to identify these bodies unless via serendipitous discovery observations. Indeed, there are some asteroids which temporarily show comet-like activity. These are among the Damocloids (C/2001 OG108 (LONEOS)), main belt asteroids (7968 ElstPizarro $=133 \mathrm{P} / \mathrm{E}-\mathrm{P})$ and Near-Earth objects $(4015$ Wilson-Harrington $=107 \mathrm{P} / \mathrm{W}-\mathrm{H})$. The important questions are: where is the pristine material in the cometary nuclei and in the asteroidcomet transition objects, do comets lose their ice or seal it in? Both the large survey projects and in-situ space missions will help to answer these questions in the near future.
\end{abstract}

Keywords. comets: general, Kuiper belt: general, minor planets, asteroids: general, Oort cloud: general, solar system: general

\section{Introduction}

Investigation of the interrelationships among the primitive small bodies can allow us to analyze the evolution of the almost unprocessed material coming from the frontiers of the solar system: from the Oort cloud and from the Transneptunian regions through Centaurs to ecliptic comets (ECs) and primitive asteroids. In addition, the Transneptunian objects, Centaurs, ecliptic comets and related asteroids are evolutionary linked.

We review new results on Damocloids (Section 2), the objects which are connected to the Oort cloud. The cometary activity of TNOs and Centaurs reveals their physicochemical characteristics and expected connections to the ecliptic comets (Section 3). The new results on Jupiter Trojan asteroids enlight their origin and possible connections to comets (Sections 4 and 5). We present the new results on the origin of ecliptic comets according to physical ground (sizes, colors) (Section 5). Furthermore we discuss the current status in the connection between main belt asteroids and cometary nuclei (Section 6). Comets and asteroids were previously thought to be two completely distinct groups of solar-system objects, with marked contrast in both physical and dynamical characteristics. A comet is operationally defined by the presence of a coma, while an asteroid has no coma. However, recent observations have shown that comets can sometimes take on asteroidal appearances and even asteroidal photometric behavior. Thus the observational distinction between comets and asteroids is not as clear cut as it once seemed. The 
possible presence of comets hidden among known asteroids forces us to reconsider the criterion by which we distinguish comets from asteroids and possibly our inventory of both comet and asteroid populations. The existence and behavior of the recurrent cometlike activity of 7968 Elst-Pizarro (133P/E-P) which orbits among the main belt asteroids pose serious problems for our understanding of comets and asteroids and how they relate to one another. We discuss the current situation of the asteroids and comets which reside in the Near-Earth and inner Earth regions (Section 7). In the end we summarize the current status and outstanding questions in the physical properties of cometary nuclei and related asteroids (Section 8).

\section{Oort cloud asteroids and asteroid-comet transition objects from the Oort cloud: the Damocloids}

Heretofore the best and effectively applicable new taxonomy of comets and other small bodies of the solar system is based on the Tisserand parameter with respect to Jupiter, $T_{J}$, in the model of Sun - Jupiter - small body circular restricted three body problem (Levison 1996). The Oort cloud associated comets with small perihelion distances, including the Halley-type comets (HTCs), are the nearly-isotropic comets (NICs) have $T_{J}<2$ (Levison et al. 2002). The Damocloids are solar system objects orbiting the Sun thought, on dynamical grounds, to be either inactive NICs or asteroids associated to the Oort cloud (Jewitt 2005). Damocloids have orbit similar to the Halley-family or NICs in general and named after 5335 Damocles. They have high orbital eccentricity and inclination, as well as most of them are without visible signs of outgassing activity, which suggest that the Damocloids are the dead or dormant nuclei of NICs (long-period comets, Asher et al. 1994). Damocloids have $T_{J}$ less than 2 , which falls to the same range as of NICs. Moreover, in correspondence with the description given by Asher et al. (1994) Jewitt (2005) has a specific definition, which restricts the Damocloids to asteroidal appearance: "a Damocloid is any point-source object having $T_{J}$ with respect to Jupiter less than or equal to 2 ". On physical ground Jewitt describes the Damocloids as inactive HTCs. In addition, to support this view, the cumulative distribution of the orbital inclination of Damocloids and Halley-type comets (HTCs) show the great similarity between the two populations Jewitt (2005).

Minor planet $1996 \mathrm{PW}$ is unusual in having the orbital characteristics of a long period comet but showing no sign of cometary activity. The discovery of $1996 \mathrm{PW}$ prompted Weissman \& Levison (1997) to examine and evaluate its possible origins, including the intriguing possibility that it is an asteroid from the Oort cloud, which is a new conception. Current models for the formation of the Oort cloud argue that most of the material there should be from the Uranus-Neptune region and thus cometary, not asteroidal, in composition. Weissman \& Levison (1997) better quantified these models and show that $\sim 1 \%$ of the Oort cloud population should be asteroids. They found that $1996 \mathrm{PW}$ has almost certainly been a resident of the Oort cloud. However, they also found it equally likely that $1996 \mathrm{PW}$ is an extinct comet or an asteroid. Although not conclusive, their results represent a significant change in our understanding of the Oort cloud, because they suggest that the ejection process sampled (i) material from as close to the Sun as the asteroid belt in the primordial solar nebula and hence (ii) much warmer formation temperatures than previously thought. They concluded that this diverse sample is preserved in the Oort cloud. Exploring of the physical properties of 1996 PW Davies et al. (1998), and Hicks et al. (2000) derived the physical parameters of this Damocloid and they found that $1996 \mathrm{PW}$ has moderately red, featureless spectra typical of the D-type asteroids, cometary nuclei, and other extinct cometary candidates. With these findings, 
1996 PW join the ranks of 3552 Don Quixote and 944 Hidalgo as established candidates for extinct comet nuclei (see also their results on the asteroid 1997 SE5).

The highly eccentric orbit of C/2001 OG108 (LONEOS) takes it closer to the Sun than the Earth, and beyond the planet Uranus. Additionally, its orbit is inclined 80 degrees with respect to the ecliptic. The object is in a Halley-family orbit, but was apparently inactive until January 2002, when it was only about 1.5 AU from the Sun and just 2 to 3 months before perihelion. Abell et al. (2003) and Fernández et al. (2003a) presented the results from multiwavelength observations of the nucleus of the unusual asteroid-comet. They observed the object in visible, near-IR, and mid-IR wavelengths near its opposition in October and November 2001, while it was still asteroidal. Thus their observations, originally intended to characterize the surface and physical properties of an unusual asteroid, were, in fact, fortuitously of a bare cometary nucleus; perhaps this nucleus is undergoing its last epoch of activity before dormancy. Very few nuclei have been studied in such detail, and even fewer nuclei that belong to comets originating in the Oort cloud. They have constrained the nucleus's size, shape, color, reflectance spectrum, albedo, and rotation period. The (near-IR) spectrum, (visible) colors, and geometric albedo most closely resemble those of a D-type asteroid. There are no absorption bands in the 0.75 to 2.4 micron range at a few percent level, though the spectrum does show a kink near 0.75 micron. The $V$-band geometric albedo of the nucleus is $0.030 \pm 0.005$, and this is well within the currently-known distribution of albedos for other active comets and extinct-comet candidates. C/2001 OG108's nucleus is both one of the largest known and one of the most slowly rotating. In addition, French (2002) reported the observation of C/2001 OG108 (LONEOS) in October of 2001: (i) image profiles consistent with those of background stars, implying no detectable cometary activity in October, and (ii) $B V R I$ colors consistent with those of inert comet nuclei and the D-class of asteroids common in the Trojan clouds.

Up to now the biggest survey of physical properties of Damocloids was performed by Jewitt (2005). In another work Fernández et al. (2005) investigated the albedos of asteroids in comet-like orbits (including Damocloids and Near-Earth Asteroids). The measured Damocloids have effective radii in the $\sim 2$ to $\sim 70 \mathrm{~km}$ range with a median effective radius of $8.4 \mathrm{~km}$. They are comparable in size to the best-observed nuclei of Jupiter-family comets (JFCs). Where measured, the geometric albedos of the Damocloids are small (0.02 to 0.04), like those of the JFC nuclei, and suggesting a dark, carbon-enriched surface composition. Jewitt (2005) presented optical measurements of 12 such objects, finding that their mean Kron-Cousins colors are $(B-V)=0.79 \pm 0.01,(V-R)=0.48 \pm 0.01$, and $(R-I)=0.48 \pm 0.01$. The normalized reflectivity spectra are generally linear, with a mean gradient $S^{\prime}=11.9 \% \pm 1.0 \%$ per $1000 \AA$. The latter is consistent with the mean $S^{\prime}=11.6 \% \pm 2.3 \%$ per $1000 \AA$ measured for the nuclei of Jupiter-family comets of ECs, a surprising result given the expected very different formation locations and dynamical histories of these two types of body. The Damocloids are devoid of the ultrared matter (with $S^{\prime} \geqslant 25 \%$ per $1000 \AA$ ) that is present on many Kuiper belt objects and Centaurs, and the mean colors of the Damocloids are inconsistent with those of the Kuiper belt objects $\left(S^{\prime}=21.1 \% \pm 1.4 \%\right.$ per $\left.1000 \AA\right)$. The data suggest that the ultrared matter, widely thought to consist of a complex organic compound processed by prolonged exposure to cosmic rays, cannot survive long in the inner solar system. Timescales for ejection or burial of ultrared matter on the nuclei of both Jupiter-family comets and Damocloids are short. Such material may also be chemically unstable to the higher temperatures experienced in the inner planetary region. Unfortunately, there are not so many Damocloids observed in detail in order to explore their physical properties (size, shape, albedo, 
color, rotational parameters, surface and thermal properties, bulk interior characteristics, cometary activity) and chemical composition hence the observation of these objects is encouraged.

\section{Cometary activity in TNOs and Centaurs: traces of volatile components}

There are a few important recent reviews on the orbital dynamics and physical characterization of Centaurs and TNOs, including their surface properties, colors, albedos, spectra, chemistry (modeling the spectra), and comet-like activity: Barucci et al. (2002), Luu \& Jewitt (2002), Schulz (2002), ESO Workshop on TNOs (Earth, Moon, and Planets Vol. 92, 2003), Barucci et al. (2004). The Centaurs and TNOs being the source of ecliptic comets, it is expected that the nature of Centaurs and TNOs be the same or very similar as that of pristine ecliptic comets, however the surface ecliptic comets altered during their evolution (Campins \& Fernández 2001, Luu \& Jewitt 2002, Jewitt 2004). The suspected conditions prevailing at the time and place of their formation support the idea that the water ice trapped large quantities of very volatile material while condensing. These hypotheses, together with the growing observations of activity of very distant comets (at heliocentric distances greater than $23 \mathrm{AU}$ ) and Centaurs, as well as with the behavior of the Pluto's atmosphere, indicate that the cometary activity in TNOs should indeed be possible. The cometary activity of Chiron inspired Brown \& Luu (1998) to investigate model comae (gas and dust) around Centaurs and KBOs. They found that the Chiron's long-lived coma cannot be a result of outburst. To observe a TNO coma requires a massive $\sim 5 \times 10^{9} \mathrm{~kg}$ dust coma, and its lifetime varies from $<2$ months to $\sim 1$ year depending on object size and heliocentric distance.

Most Centaurs are inactive but a few display cometary activity: 95P/Chiron (2060 Chiron), 29P/Schwassmann-Wachmann 1, 39P/Oterma, 165P/2000 B4 (LINEAR), 166P/2001 T4 (NEAT), C/2001 M10 (NEAT), and 167P/2004 PY42 (CINEOS). We note that $29 \mathrm{P}$ with $q=5.7 \mathrm{AU}$ and $a=6.0$ AU satisfies the definition of Jewitt \& Kalas (1998) for Centaurs but that it has $T_{J}=2.983$ (while for Centaurs $T_{J}>3$ ). In addition, there is a nomenclature problem in the designation of active Centaurs with cometary identification: the cometary provisional or periodic comet identification is preferred, rather than their names (Green 2005). Recently Bauer et al. (2003) presented the results of optical observations of 166P/2001 T4 (NEAT) in 2001 and 2002. Coma was present for each observations but the activity level was variable. Dust production rate was between $\sim 10^{-2}$ and $20 \mathrm{~kg} \mathrm{~s}^{-1}$, comparable to active Jupiter-family comets (cf. A'Hearn et al. 1995).

Aside the well known cometary activity in Centaurs a few years ago there were observations which are interpreted as direct evidences of TNO cometary activity. Most notably, Fletcher et al. (2000) (see Delahodde et al. 2000) announced the detection of a coma around 1994 TB, using HST. Hainaut et al. (2000), Delahodde et al. (2000) obtained a detailed portrait of 1996 TO66 using VLT: a dramatic change of that object's rotational lightcurve between 1997 and 1998 is interpreted as the signature of a cometary outburst.

Meech et al. (2003) searched for cometary activity in KBO (24952) 1997 QJ4 using the Subaru 8-m telescope. There is a large color diversity among the KBOs. The neutral blue colors with respect to the Sun can be explained by the possible surface outgassing (cometary) activity (it is the most plausible explanation but there are other causes of the surface blueing during the evolution of the outer solar system objects). They selected this target because of its intrinsically blue color $(V-R)=0.296$. They placed sensitive upper limits on the dust production rate from the object at $Q<0.01 \mathrm{~kg} \mathrm{~s}^{-1}$. 
Surveying of the cometary activity of the faint objects in the outer solar system is usually a very difficult task despite of using large telescopes. For example the Scattered Disk object (29981) 1999 TD10 was observed simultaneously in the $R, J$ and $H$ bands in September 2001, and in $B, V, R$, and $I$ in October 2002 by Mueller et al. (2004). But their observations at the same time, with better $\mathrm{S} / \mathrm{N}$ and seeing, show no evidence of a coma, contrary to the claim by Choi et al. (2003). Moreover, Rousselot et al. (2003) also did not observed cometary activity on 1999 TD10.

Regarding the cometary activity of Centaurs and TNOs obviously volatile compounds are needed. Brown \& Koresko (1998) reported the detection of the 1.5 and 2.0 micron $\mathrm{m}$ absorption bands due to water ice in the near-infrared reflection spectrum of the Centaur 10199 Chariklo (1997 CU26). The water ice bands are weaker than those detected on the surface of any other solar system body; the spectrum is well fit with a model surface consisting predominantly of a neutral dark absorbing substance with only $\sim 3 \%$ a real coverage of water ice. Luu et al. (2000) reported the detection of water ice in the Centaur 2060 Chiron, based on near-infrared spectra (1.0-2.5 micron). The appearance of this ice is correlated with the recent decline in Chiron's cometary activity: the decrease in the coma cross section allows previously hidden solid-state surface features to be seen. They predicted that water ice is ubiquitous among Centaurs and Kuiper belt objects, but its surface coverage varies from object to object and thus determines its detectability and the occurrence of cometary activity. Independently, Foster et al. (1999) reported the presence of the water ice 2.03 micron spectral feature in the reflectance spectrum of Chiron and several other Centaurs. In the frame of the ESO Large Program on Transneptunian Objects and Centaurs Boehnhardt et al. (2003) obtained 12 spectra in the visible region and nine of them for which they obtained also near infrared spectra up to 2.4 microns. The principal reported results obtained are, including possible detection of water ice: (i) a wide range of visible slopes, (ii) evidence for surface variations on $2001 \mathrm{PT} 13$, and ( iii) possible detection of few percent of water ice (1999 TC36), 2000 EB173, 1999 DE9, 2001 PT13, 2000 QC243, 1998 SG35).

More accurate and realistic models are important in understanding the surface properties and comet-like activity in the outer solar system objects. In addition, combining the visible and infrared observations, as well as using an adequate thermal model the effective radius and albedo can be determined. Fernández et al. (2003b) measured the mid-infrared thermal continuum from inactive 8405 Asbolus and active 2060 Chiron. Using simple thermal models they found that Chiron has a variable dust coma. The surface heterogenity of 10199 Chariklo (1997 CU26) was taken into account in the models developed by Dotto et al. (2003) and compared with the near-infrared observations. They used tholins, amorphous carbon and water ice and they confirmed the presence of water ice on the surface of this Centaur as it was detected earlier by Brown \& Koresko (1998). A new surface thermal model of Centaurs was developed by Groussin et al. (2004) who analyzed visible, infrared, radio and spectroscopic observations of 2060 Chiron (95P/Chiron) in a synthetic way to determine its physical properties. Infrared observations at 25, 60, 100 and 160 micron (i.e., covering the broad maximum of the spectral energy distribution) obtained with the Infrared Space Observatory Photometer (ISOPHOT) in June 1996 when Chiron was near its perihelion are analyzed with a thermal model which considers an intimate mixture of water ice and refractory materials and includes heat conduction into the interior of the nucleus. They found that the observed spectra of Chiron can be fitted by a mixture of water ice $(\sim 30 \%)$ and refractory $(\sim 70 \%)$ grains, and that this surface model has a geometric albedo consistent with the above value. They also analyzed the visible, infrared and radio observations of Chariklo (1997 CU26) and concluded 
that a mixture of water ice $(\sim 20 \%)$ and refractory $(\sim 80 \%)$ grains is compatible with the near-infrared spectrum and the above albedo.

First evidence for the presence of water ice was detected in TNO 1996 TO66 in the NIR spectra taken with the Keck telescope Brown et al. (1999). The published Keck NIR spectra of 1996 TO66 obtained almost simultaneously with 1998 data obtained by Hainaut et al. (2000), showing the presence of water ice on that object, further reinforcing the conviction of its cometary nature.

Later, Bockelée-Morvan et al. (2001) searched for rotational lines of CO in Pluto/Charon (the largest known bodies among the KBOs), other KBOs, and Centaurs at radio wavelengths, i.e., the $\mathrm{CO}$ as a supervolatile compound was looked for which can drive the outgassing activity at large heliocentric distances beyond the heliocentric distance limit of the water ice sublimation activity. None of the Centaurs or Kuiper belt objects were detected in $\mathrm{CO}$. The $\mathrm{CO}$ production rate upper limit obtained for Chiron $\left(3-5 \times 10^{27} \mathrm{~mol} \mathrm{~s}^{-1}\right)$ over $1998-2000$ years is a factor of 10 lower than the CO production rate derived from the marginal CO detection obtained in June 1995 by Womack \& Stern (1999), using same modeling of CO emission. Upper limits obtained for other Centaurs are typically $\sim 10^{28} \mathrm{~mol} \mathrm{~s}^{-1}$, and between 1 and $5 \times 10^{28} \mathrm{~mol} \mathrm{~s}^{-1}$ for the best observed KBOs. Bockelée-Morvan et al. (2001) concluded that the comparison between these upper limits and the CO outgassing rates of comet C/1995 O1 (Hale-Bopp) measured at large distances from the Sun shows that Centaurs and KBOs underwent significant COdevolatilization since their formation.

Campins \& Fernández (2001), Licandro et al. (2002), Licandro et al. (2003) presented the results of their near-infrared spectroscopic program of TNOs, Centaurs and comet nuclei. TNOs, Centaurs, and Jupiter-family comets are three intimately related populations of minor planet bodies originated in the outer solar system. They probably contain some of the least modified materials remaining from the protosolar nebula. The study of their physical properties and evolution provide invaluable cosmogonical information. Near-infrared spectroscopy is a diagnostic method for remote determination of the surface composition of these objects. In addition to the already published spectra of 38628 Huya and 20000 Varuna, and 28978 Ixion (Licandro et al. 2003 amd references therein) and 124P/Mrkos they presented new spectra of the TNOs 38628 Huya, 50000 Quaoar, and 1999 TC36, and the Centaurs 8405 Asbolus, (54598) 2000 QC243, 32532 Thereus, 31824 Elatus, and 2002 PN34. Water ice absorption bands are present in the spectra of several objects. Evidence of surface inhomogeneities is also presented, in particular in the case of 32532 Thereus that was observed during an almost complete rotation. The results are discussed in the framework of the possible resurfacing mechanisms proposed (space weathering, coma activity and collisions).

Fornasier et al. (2004a) obtained visible and near infrared spectra of the TNO 90482 Orcus (2004 DW), a few days after its discovery, at the Telescopio Nazionale Galileo (TNG). 90482 Orcus belongs to the plutino dynamical class and has an estimated diameter of about $1600 \mathrm{~km}$, that makes it one of the largest KBO. Data clearly show the 1.5 and 2 micron bands associated to water ice, while the visible spectrum is nearly neutral and featureless. To interpret the available data the best fit model of the surface composition of 90482 Orcus (2004 DW) contains two different mixtures of organics (Titan tholin and kerogen), amorphous carbon and water ice.

During the last years water ice has been reported in a handful of objects in the outer solar system, but most appear spectrally featureless. Most recently Jewitt \& Luu (2004) published their infrared spectroscopic observations of the large KBO 50000 Quaoar, which reveal the presence of crystalline water ice and ammonia hydrate. Crystallinity indicates that the ice has been heated at least $110 \mathrm{~K}$. Both ammonia and water ice should be 
destroyed by energetic particle irradiation on a timescale of about $10^{7} \mathrm{yr}$. They concluded that Quaoar has been recently resurfaced, either by impact exposure of previously buried (shielded) ices or by cryovolcanic outgassing, or by a combination of these processes.

Another possibility to preserve water in small bodies are the aqueously altered minerals. de Bergh et al. (2004) obtained visible and near-infrared spectra, as well as photometric data, for two TNOs, (47932) 2000 GN171 and 38628 Huya (2000 EB173), which belong to the dynamical class of plutinos in 2001 and 2002. The features detected in the visible spectra of the two objects are tentatively attributed to the presence of iron oxides or phyllosilicates at the surfaces of the two objects. There are differences between the April 2001 and May 2002 visible spectra, which are attributed to spatial variations at the surfaces of the objects. They proposed the possibilities for aqueous alteration in TNOs, after reviewing what we know about the presence of aqueously altered minerals (silicates) in other small bodies of the solar system. They suggested further studies monitoring the rotation of these two objects are highly desirable.

\section{Jupiter Trojan asteroids and comets}

The most recent reviews and results on the dynamical characteristics of Trojans are of Beaugé \& Roig, Marzari et al. (2002), Karlsson (2004), Morbidelli et al. (2005), as well as on the physical properties are of Barucci et al. (2002), Fernández et al. (2003b), Jewitt et al. (2000), Licandro et al. (2002), Jewitt (2004), Bendjoya et al. (2004), Emery \& Brown (2003), Emery \& Brown (2004), Fornasier et al. (2004b).

The observed physical properties (shape, color, albedo) of the Jupiter Trojans are formally indistinguishable from those of the cometary nuclei (Jewitt \& Luu 1990, Fernández et al. 2003c). As Jewitt (2004) warned: this suggests but does not prove an intriguing compositional similarity between the two classes of body, at least at the surface level where irradiation and solar heating may play a role. No ices have been spectroscopically detected on the Trojans (Jones et al. 1990, Luu et al. 1994, Dumas et al. 1998, Emery \& Brown 2003). Bendjoya et al. (2004) observed spectra of 34 Jupiter Trojans with the Danish 1.54-m telescope at ESO. They found that large majority of the objects of the sample have been observed to belong to the D taxonomic class, but they found also objects of P- and C-type. In two cases, they found also evidence of blueish spectral trends. These data are important, since they allow us to substantially enlarge the whole data set of available Trojan spectra. Moreover, the observations are also confirmed the lack of absorption features in the visible (Licandro et al. 2002, Fornasier et al. 2004), and in the near-infrared (Emery \& Brown 2000). The collisional evolution is important in the Trojan swarms. Most recently Melita et al. (2005) investigated the connections between the physical and dynamical characteristics of the Jupiter Trojans. They found that the moderately-red and stable objects are the close descendants of the primordial Trojan precursors. The color of the unstable Trojans are uniformly distributed over a wide range the spectral slope $\left(S^{\prime}\right)$ from 0 to about $15 \%$ per $10^{3} \AA$.

Beneath their refractory mantles, however, the Trojans may be ice rich (Jewitt 2004), so it is difficult to show outgassing activity. Unfortunately, they are too cold to measurably sublimate even if ice is present in the near surface regions. Very recently Emery \& Brown (2004) modeled the observed spectra of 17 Jupiter Trojans and they estimated that the surfaces of these asteroids contain at most a few wr\% of water ice and no more than 10-30 wr\% of hydrated silicates. These findings are in agreement with those of Cruikshank et al. (2001) who estimated $3 \mathrm{wr} \%$ water ice and $40 \mathrm{wr} \%$ hydrated silicate content of the surface of 624 Hektor. This indicates that hydrous minerals might be present in the surface material and remain undetected with the quality of near-infrared spectroscopic data what now is available. So, there is some hope to find trace of water 
in the Jupiter Trojans. In addition, the Trojans have very low albedos, consistent with the C-, P-, and D-type asteroids and with many of the small outer satellites of Jupiter (Jewitt et al. 2004). The spectral features at 0.7 and 3 micron in the spectra of many low-albedo $\mathrm{C}, \mathrm{G}$, and $\mathrm{F}$ class main belt asteroids are associated with hydrous minerals. Hydrous minerals might associate with the heating of interior ice in an earlier epoch, or they might indicate the incorporation of materials that were serpentized by other processes. The highly volatile molecules $\mathrm{N}_{2}, \mathrm{CO}$, and $\mathrm{CH}_{4}$ were not efficiently trapped and incorporated into the planetesimals formed at the heliocentric distance of Jupiter, but $\mathrm{NH}_{3}$, refractory organics, and compounds of other heavy elements were trapped (see Barucci et al. 2002 and references therein).

Regarding the connection between Jupiter Trojan asteroids and comets the first conceptions are based on celestial mechanics. The similarities observed between the physical properties of the nuclei and those of the Trojan asteroids partially explained by any of the capture hypotheses. Temporary captures of comets at Lagrangian points are known to have occurred in the recent past, moreover, the escapes from the L4 and L5 clouds can supply the JFCs (Rabe 1972). Recently, the formation of the trojan swarms are explained in detail by Marzari \& Scholl (1998a), Marzari Scholl (1998b), Fleming \& Hamilton (2000), Marzari et al. (2003), and Morbidelli et al. (2005). The existence of objects can be captured to the 1:1 MMR to Jupiter was proven recently by Karlsson (2004) who showed by performing extensive numerical intergrations that there are a few objects which temporary reside in Jupiter Trojan orbit. In addition, the numerical models by Morbidelli et al. (2005) pointed out that there are effective capturing processes to lock outer solar system objects like TNOs, Centaurs in Jupiter Trojan orbits, i.e., the chaotic capture mechanism was effective in the early solar system. The connection between the Jupiter Trojans and comets was revisited by Marzari et al. (1997), i.e., the Jupiter Trojans are considered as a source region of Jupiter-family comets. They concluded that the Jupiter Trojans may contribute to the comet population through dynamical instabilities and collisional ejection. Once removed from the vicinity of the Lagrangian $\mathrm{L}_{4}$ and $\mathrm{L}_{5}$ points, they quickly lose dynamical traces of their origin. Quantitatively, there are too few Jovian Trojans to supply more than $\sim 10 \%$ of the flux of Jupiter-family comets (Marzari et al. 1997, Jewitt et al. 2000).

\section{Origin of the ecliptic comets based on comparison of their size distribution and colors with those of other primitive small bodies}

Our recent knowledge is that the TNOs are dynamically related to the Centaurs and the Jupiter-family group of ecliptic comets, even the progenitors of the Centaurs are the TNOs, as well as the parent bodies of the ecliptic comets can be found among either the Centaurs or SDOs. The idea that TNOs may be the source of both, Centaurs and ecliptic comets, is widely supported by dynamical considerations even recently by physical observations (see the results and reviews by Stern 1995, Farinella \& Davis 1996, Duncan \& Levison 1997, Luu \& Jewitt 2002, Schulz 2002 and references therein). Simply due to the observational constraints the available data are statistically limited in the studies of the connections and evolutionary links between different classes of primitive small bodies therefore the comparison of the size distributions and the surface colors are hitherto the best methods.

\subsection{Size distribution of cometary nuclei}

First we discuss the size distribution of the cometary nuclei, especially the size distribution of the ecliptic comets, which is based on the data sets selected and analized by Lamy 
Table 1. Power exponents of the Cumulative Size Distribution (CSD) of the ecliptic comets and other small body pouplations (Lamy et al. 2004)

\begin{tabular}{lcl}
\hline \multicolumn{1}{c}{ Population } & CSD & \multicolumn{1}{c}{ Reference } \\
\hline ECs & & \\
ECs & $2.65 \pm 0.25$ & J. Fernández et al. $(1999)$ \\
ECs & $1.6 \pm 0.1$ & Lowry et al. $(2003)$ \\
ECs & $2.5^{*}$ & Meech et al. $(2004)$ \\
ECs & $1.59 \pm 0.03$ & Lowry \& Weissman $(2003)$ \\
ECs & $1.79 \pm 0.05$ & Weissman (private comm., 2003) \\
KBOs $(r>20 \mathrm{~km})$ & $1.9 \pm 0.3$ & Lamy et al. $(2004)$ \\
& 3.45 & Gladman et al. $(2001)$ \\
Centaurs & $3.20 \pm 0.10$ & Larsen et al. $(2001)$ \\
& $3.15 \pm 0.10$ & Trujillo et al. $(2001)$ \\
ECs + "cometary" NEOs & $2.70 \pm 0.35$ & Larsen et al. $(2001)$ \\
Near-Earth objects & 3.0 & Sheppard et al. $(2000)$ \\
& $1.6 \pm 0.2$ & Lamy et al. $(2004)$ \\
Main belt asteroids & $1.75 \pm 0.10$ & Bottke et al. $(2002 \mathrm{~b})$ \\
Jupiter Trojans $(2.2 \leqslant r \leqslant 20 \mathrm{~km})$ & 1.96 & Stuart (2001) \\
Jupiter Trojans $(r \geqslant 42 \mathrm{~km})$ & $1.25-2.80$ & Jedicke \& Metcalfe $(1998)$ \\
& $2.0 \pm 0.3$ & Jewitt et al. $(2000)$ \\
\hline
\end{tabular}

${ }^{*}$ From Monte Carlo simulations after truncation at small radii.

et al. (2004). These represent the largest data sets ever assembled. Figures 1 (left and right panels) present the distribution functions of the volume equivalent effective radius of ECs and NICs. The histograms show several structures, which most likely result from the limited statistics in the data set. The apparent roll-off in the number of small cometary nuclei is very likely an observational selection effect (i.e. incompleteness of the data sample, smaller nuclei are simply harder to detect). A similar effect is often encountered with flux-limited surveys, but additional mechanisms cannot be excluded, for example the hypothesized large population of very small EC nuclei (Brandt et al. (1996)) and the observed sungrazing fragments of split comets (Biesecker et al. 2002).

Cumulative size distribution function (CSD) is used, which is robust and less prone to artifacts: $N_{S}\left(>r_{n}\right)$, where $N_{S}$ is the number of nuclei larger than radius $r_{n}$ is represented by power law:

$$
N_{S}\left(>r_{n}\right) \propto r_{n}^{-q_{S}}
$$

where $r_{n}$ is the radius of the nucleus, and $q_{S}$ is the power-exponent of the size distribution function. Figure 1 (right panel) displays the CSD of the 13 nearly-isotropic cometary nuclei whose effective radii have been determined. Also plotted is the CSD of this population augmented by the 12 asteroidal objects thought to be dormant or extinct NICs on the basis of their Tisserand parameters. Owing to the poor statistics, Lamy et al. (2004) did not attempt to fit power laws to the observed CSDs. Results of $q_{S}$ for various minor body populations in the solar system are summarized in Table 1.

If the ECs are collisional fragments of TNOs (Stern 1995, Farinella \& Davis 1996), then the theoretical value $q_{S}=2.5$ for a collisionally relaxed population (Dohnanyi 1969 ) is expected. In reality, the question is probably more complex. The simple model of Dohnanyi (1969) applies to a population of self-similar bodies having the same strength per unit mass. Several groups have attempted to improve this simplified assumption, with 

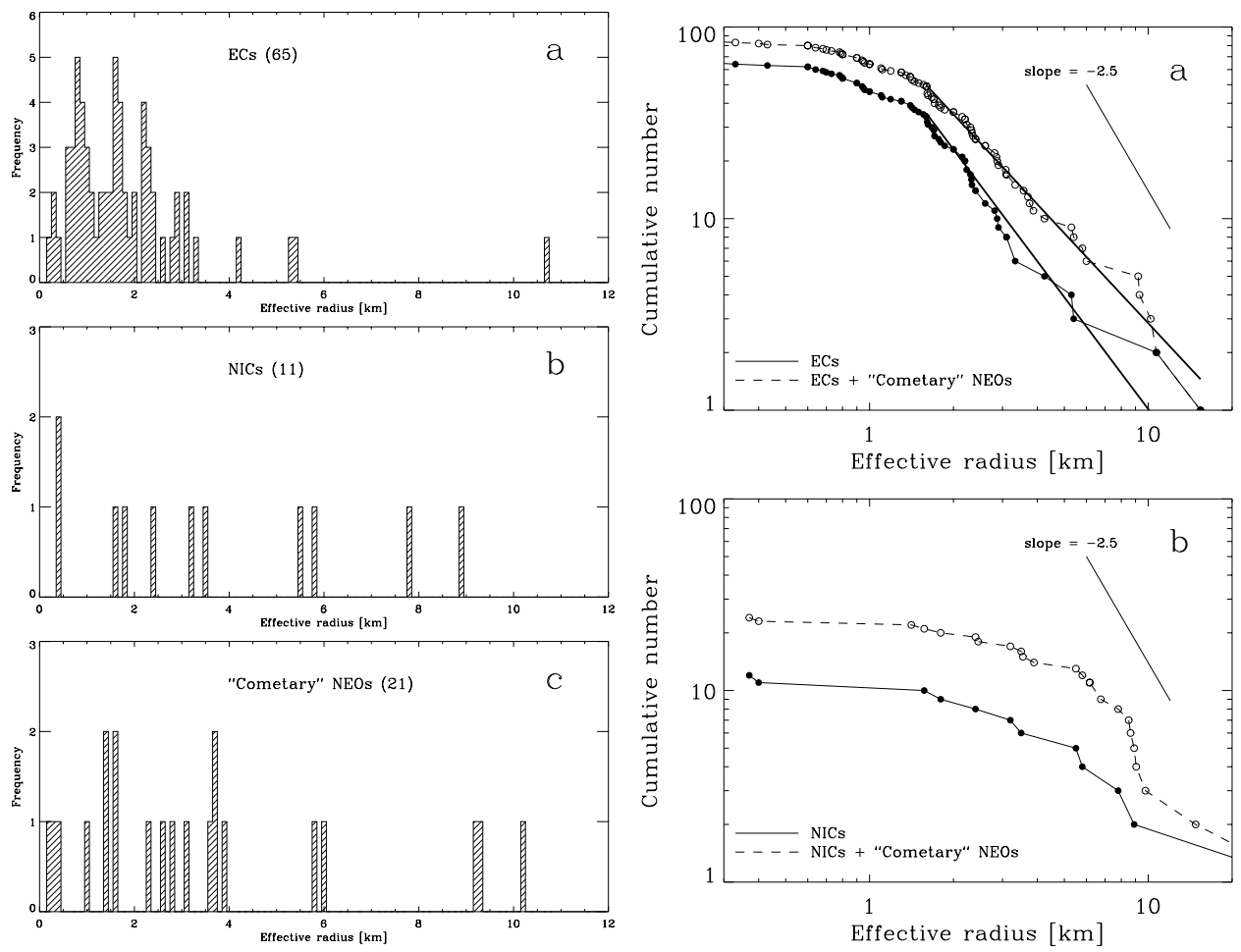

Figure 1. Left panel: Size distribution of the cometary nuclei. Distribution of the effective radius for ecliptic comets (ECs) (a), for nearly-isotropic comets (NICs) (b), for cometary Near-Earth objects $(c)$. Note that the largest nuclei are excluded to allow legibility of the historgram at small sizes. Numbers of the objects in the samples are indicated in brackets in each panels. Right panel: Cumulative size distribution of the nuclei of ecliptic comets (ECs) (a), nearly-isotropic comets (NICs) and cometary Near-Earth objects $(b)$ are represented by solid circles while the open circles apply to the population augmented by the "cometary" NEOs. The two solid lines in panel $(a)$ correspond to optimum power-law fits according to the Kolmogorov-Smirnov tests, from the cut-off radius $1.6 \mathrm{~km}$ up to the largest bodies. Figures from Lamy et al. (2004).

O'Brien \& Greenberg (2003) presenting the most comprehensive results on steady-state size distributions for collisional populations. In the range of sizes of interest for cometary nuclei, the size distribution of fragments is wavy, and oscillates about the distribution of a population evolved under pure gravity scaling. The differential size distribution of such a population is characterized by a power law with an exponent of -3.04 . This translates into $q_{S}=2.04$ using our notation for the cumulative distribution. Regarding the size distribution of the KBOs, the progenitors of the Centaurs and ECs, most recently Pan \& Sari (2005) revisited the problem of the KBO size distribution. They found that the power exponent $q_{S}=2$, which is in agreement with the findings of Bernstein et al. (2004), namely the KBOs smaller than $\sim 40 \mathrm{~km}$ are effectively strengthless. This does not mean that the ECs are directly related to the KBOs according to the similarity of their size distribution only since there are many size altering processes in the KBO Centaur - EC evolutionary link (see below).

On the other hand, non-collisional fragmentation (i.e. splitting) is frequent among comets (see Boehnhardt 2004), and nuclei are progressively eroded by their repeated passages through the inner part of the solar system, so that we are certainly not observing a primordial, collisionally relaxed population of TNO fragments (Lowry \& Weissman 
(2003) Samarasinha (2003)). Mass loss may therefore significantly distort the size distribution of nuclei, particularly at the low end. Moreover there is a difficulty in modeling the evolution of the nuclei due to the chaotic nature of the orbital evolution of ecliptic comets.

In summary, for ECs the $q_{S}$ could be as small as $\sim 1.6$ and as large as $\sim 2.5$, with a preferred value of $\sim 2.0$. However, we will quote $q_{S}=1.9 \pm 0.3$ because that is our result for the CSD that includes all the ECs for which reliable data have been obtained. Table 1 shows that this result is intermediate between those of Lowry et al. (2003) $\left(q_{S}=1.6 \pm 0.1\right)$ and Lowry \& Weissman $(2003)\left(q_{S}=1.59 \pm 0.03\right)$, recently revised to $q_{S}=1.79 \pm 0.05$; Weissman, private communication (2003), on the one hand, and Fernández et al. (1999) $\left(q_{S}=2.65 \pm 0.25\right)$, on the other hand.

In addition, the population of asteroidal objects thought to be dormant or extinct comets are considered, on the basis of their Tisserand parameters, or their association with meteor streams. The cometary origin of these NEOs is still highly speculative, and many of them may be asteroids coming from the outer regions of the asteroidal belt, including the Hilda group and Jupiter Trojans (Fernández et al. 2002). Selection effects are also different from those of the ECs, and any future unbiasing should reflect these differences. For the purpose of the present exercise, we considered 21 "cometary" NEOs that can be associated with ECs, and whose sizes have been determined (Table 1), thus bringing the data base to 86 objects. The "cometary" NEOs tend to be larger on average than the ECs, thus significantly filling the $2-10 \mathrm{~km}$ radius range, but flattening the CSD simply because they are more of them at larger sizes; indeed, we found $q_{S}=1.6 \pm 0.2$, $P_{K S}$ reaching 0.85 when including these NEOs.

The power exponent of the CSD of KBOs is quite large, $q_{S}=3.15-3.45$, but strictly applies to objects with $r_{n}>20 \mathrm{~km}$. It is not clear whether this value extends down to smaller sizes to allow a meaningful comparison with ECs. In fact, it has been suggested that KBOs follow a broken power law with the larger objects $\left(r_{n} \gtrsim 50 \mathrm{~km}\right)$ retaining their primordial size distribution with the above value of $q_{S}$, while the smaller objects represent collisional fragments having a shallower distribution (e.g., Davis \& Farinella 1996), which could then be rather similar to that of the ECs. The power exponent of the CSD of Centaurs, $q_{S}=2.7-3.0$, is also larger than that of the ECs. However, the statistics are rather poor, and we found that, from the data of 9 Centaurs reported by Barucci et al. (2004), it is very difficult to fit a power law to the observed CSD: the exponent can take any value, from 3.1 down to 1.2 , depending on the imposed cut-off at small sizes.

The CSD of ecliptic comets is beginning to look remarkably similar to that of NearEarth objects: note the result of Stuart (2001), $q_{S}=1.96$ which is essentially identical to our value. For the main-belt asteroids, size distributions are so well-defined that changes in the power exponent can be recognized in different size regimes (see the details in (Jedicke \& Metcalfe 1998), and we have simply indicated the ranges. NEOs and mainbelt asteroids are thought to be collisionally dominated populations, yet they have power exponents significantly different from the canonical value of $q_{S}=2.5$ obtained by Dohnanyi (1969).

For the Jupiter Trojans, the size distribution exhibits a bimodal structure, i.e., the power exponent can be recognized in two different size regimes (see the details in Jewitt et al. 2000), and the separating size is $\sim 40 \mathrm{~km}$ (Table 1). The small Trojan asteroids are collisionally produced fragments of large bodies (Marzari et al. 1997, Jewitt et al. 2000).

A final comparison is that with the CSD of the fragments of comet D/1999 S4 (LINEAR): from water production rates measured following its breakup, Mäkinen et al. (2001) 

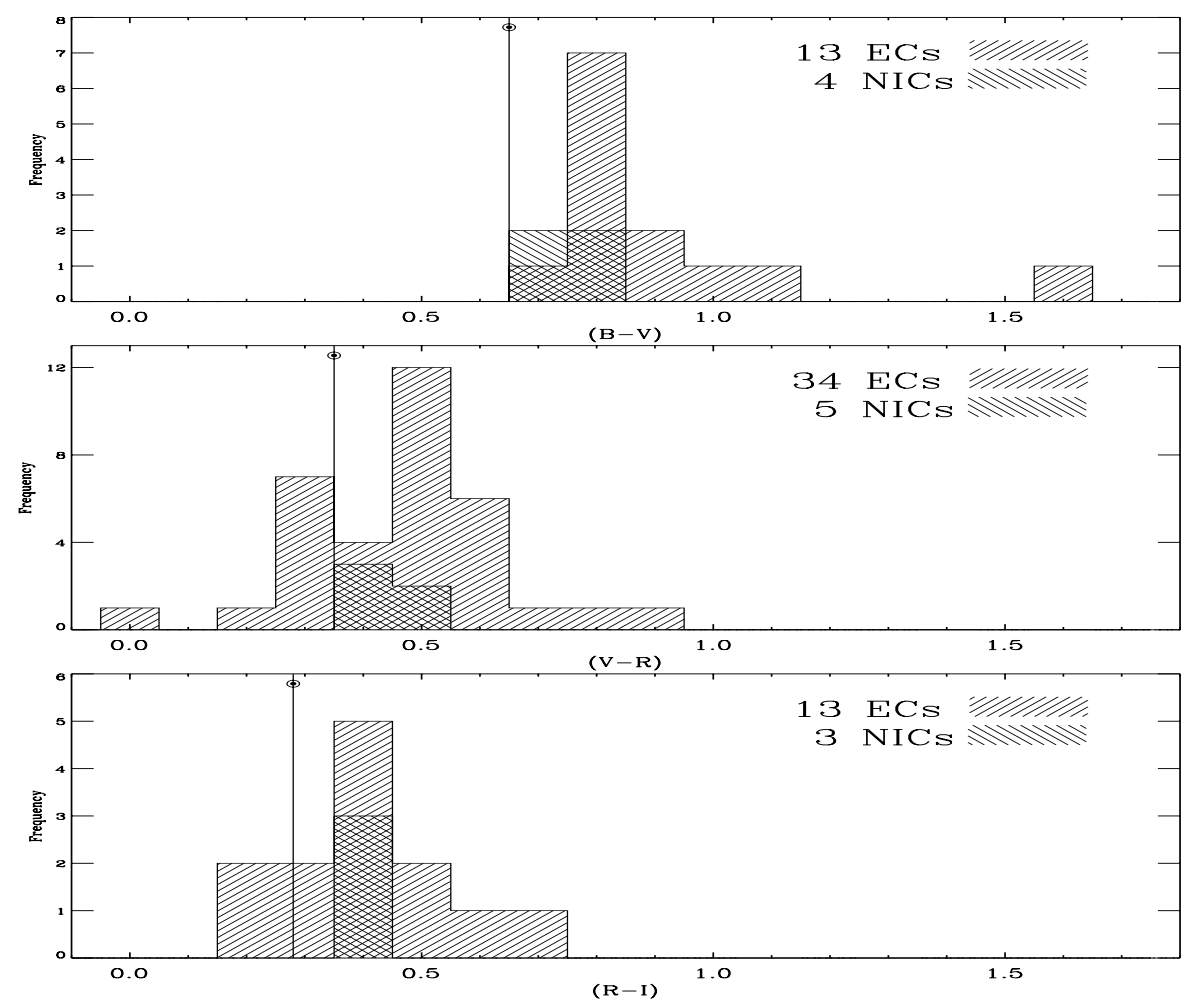

Figure 2. Distribution of the color indices for the cometary nuclei observed with the Hubble Space Telescope: $(B-V)$ (top), $(V-R)$ (middle), and $(R-I)$ (bottom). Numbers of the objects in the samples are indicated in each panels. Figure from Lamy \& Toth (2005).

found that the measurements could best be explained by a fragment size distribution having $q_{S}=1.74$, which is within the range we estimate for the ECs.

The question of the size distribution of ECs at the lower end, $r_{c}<1.6 \mathrm{~km}$, remains totally open. The possible influence of both observational and evolutionary biases has been mentioned already, but a real depletion cannot be excluded. Indeed, the depletion of small nuclei is supported by the measurements of crater distributions on several airless bodies of the solar system, where cratering from comets is believed to dominate, e.g., Europa (Chapman et al. 1997) and Ganymede and Callisto (Zhanle et al. 2001).

\subsection{Colors of the cometary nuclei and other primitive small bodies}

Lamy \& Toth (2005) extensively explored the statistical properties of the broad-band colors of cometary nuclei and reported color data for 23 active cometary nuclei detected with the Hubble Space Telescope using their well-proven method of nucleus-coma separation. In addition, they supplied the comet nucleus color data with data observed by others, as well as built a color data base of currently available colors of Centaurs, TNOs and other primitive minor bodies.

Figure 2 displays the histograms of the three color indices $(B-V),(V-R)$ and $(R-I)$ constructed with a bin size of 0.1 . The gaps must not be overinterpreted as suggesting different groups but most likely result from the (still) limited coverage, particularly for the $(B-V)$ and $(R-I)$ colors. The meager color data we presently have on the nuclei of NICs indicate less diversity of colors and the absence of blue nuclei. It will be interesting to see whether those trends are confirmed when the sample of NICs will expand. 
Lamy \& Toth (2005) presented implications of colors for searching for the progenitor groups among the primitive minor bodies to determine the "parent-child" relationships. In order to refine the progenitor searching all the Centaurs were considered as single group, moreover, the Centaurs were split into "Centaurs I" (red) and "Centaurs II" (blue) subgroups according to their color bimodality discovered by Peixinho et al. (2004). Additionally, the classical KBOs (CKBOs) are split into the low- and high-inclination subgroups (CKBO-LI and CKBO-HI). Use of Kolmogorov-Smirnov (K-S) probability for the cumulative distribution functions of one or two color index samples the parentchild connections are summarized as follows. To take into the blueing in the evolution of primitive small bodies the color shift was applied in the K-S tests to catch the perfect fit of the compared color distributions. For the global population of Centaurs, the K-S test favors the Plutinos as progenitors and rules out the CKBO-LI subgroup. The connection disappears when considering the two populations of Centaurs (Centaur I and II). In order of importance (K-S probability), the connections are Centarur - EC, SDO - EC, and Plutino - EC but the probabilities remain low. The first connection becomes less obvious when Centaurs are split. Significant color shifts are required to boost the probabilities to creditable levels. The probabilities of the EC - dead comets connection are low and again can only be increased by significant shifts.

The present global picture of colors of primitive bodies of the solar system could be explained along the following lines. The coherent and progressive color shifts are consistent with the following non-unique scenarios. CKBO-LI formed in-situ beyond the orbit of Neptune while CKBO-HI, Plutino and SDOs formed inside this orbit, a picture remarkably consistent with that proposed by Gomes (2003). SDOs could have formed at slightly shorter distances than CKBO-HI and Plutinos, possibly explaining their more efficient scattering. Centaurs remains a puzzle. As a single group, the colors suggest they come from Plutinos. As a dual group, Centaurs I could be composed of TNOs (CKBOs, Plutinos, SDOs) and ultra-red bodies from the inner Oort cloud while Centaurs II have already visited the inner solar system and lost their red organics; their origin is unclear on the basis of colors alone. Ecliptic comets lost part of their red organics, most likely thermal alteration, the end-state being reached by the candidate dead comets. This process involves different red compounds having different thermal instabilities and different optical properties. The large range of colors that KBOs, Centaurs and ECs share results from a common large diversity in their composition. It implies limited mixing in the outer part of the protoplanetary disk leading to accretion of unhomogeneous planetesimals.

As a final remark, we stress that the number of objects for which we have $B V R I$ colors remain small, not to say extremely small so that the presently available data sets may simply be not representative of the reality. Recent controversies often resulting from biased data sets give the lesson that we must remain prudent in our interpretations.

\section{Connections between comets and main belt asteroids}

There are comets, which orbit in the main asteroid belt. The most abundant such a comet population resides in the Hilda asteroid zone in the outer main belt. The most prominent example for this comet population is D/Shoemaker-Levy 9 prior to its capture by Jupiter (Chodas \& Yeomans 1995, Tancredi \& Sosa 1996). On its possible pre-capture orbit this comet dwelt in the Hilda asteroid zone before, i.e., its probable pre-capture orbits overlap a group of known comets, referred to as quasi-Hildas by Kresák (1979). In addition, Kresák (1979) presented an inventory of other cometary groups which reside in the main asteroid belt. Most recently, Toth (2005a) updated the inventory of the 


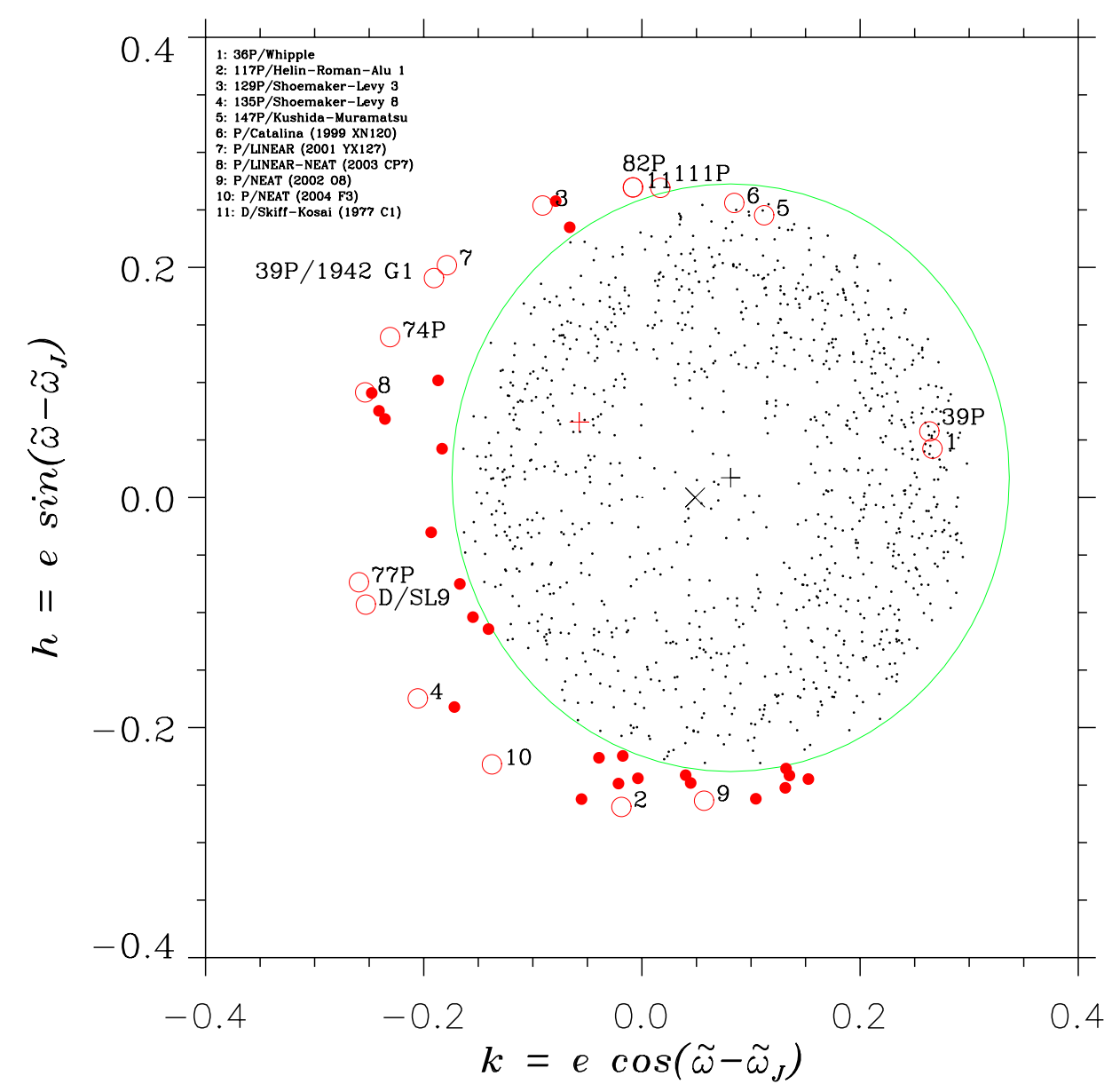

Figure 3. Location of the Hilda asteroid family members and the quasi-Hilda comets in the Lagrangian elements $\left(e_{x}, e_{y}\right)=(k, h)$ plane is displayed. The Hilda asteroids are shown with black dots and the quasi-Hilda comets are represented by red open circles. The newly identified Hilda asteroid outliers are highlighted with red dots. The mean value of the $k$ and $h$ coordinates of the samples of Hilda asteroids and quasi-Hilda comets are shown with a plus sign $(+)$. Location of Jupiter is shown with a cross $(\times)$. Figure from Toth (2005a).

quasi-Hilda group of possibly comets searching for outliers in the Lagrangian elements space (Fig. 3). New members (11) of the ecliptic comets and Hilda outliers (24) were found. However, there is no observation to explore the physical characteristics of these objects but their orbits belong to the quasi-Hilda comet group. Among these objects can be dormant or extinct cometary nuclei. Physical observations of the Hilda asteroids and quasi-Hilda comets (candidate quasi-Hilda comets) are needed.

There are asteroids which display temporary comet-like activity. These are the asteroidcomet transition objects which can be found among the small body populations in the solar system (Weissman et al. 2004, Jewitt 2004, Jewitt 2005). There are two distinguished examples for this behavior: 4015 Wilson-Harrington and 7968 Elst-Pizarro with showing temporary dust tail and trail (see the history of 107P described by Fernández et al. 1997, and 133P described by Toth 2000, and Hsieh et al. 2004, as well as general reviews by Weissman et al. 2002, Jewitt 2004). These two objects are also catalogized 
as comets: 107P/Wilson-Harrington (Fernández et al. 1997, Coradini et al. 1997, Jewitt 1996, Jewitt 2004), and 133P/Elst-Pizarro (hereafter E-P in short) but they have typically asteroid orbit with value of Tisserand parameter with respect to Jupiter 3.084 for 107P and 3.184 for E-P.

Elst-Pizarro shows recurrent comet-like activity but it resides in the Themis-zone of the main asteroid belt $\left(a=3.158 \mathrm{AU}, e=0.165, i=1.38^{\circ}\right)$ (Boehnhardt et al. 1998, Lien 1998, Toth 2000, Weissman et al. 2002, Hsieh et al. 2004). The hypothesis of recent impact event was among the first explanations of the dust trail (or tail) of E-P, which excavated or activated the surface material and generated the dust mass-loss (Boehnhardt et al. 1998, Lien 1998, and Toth 2000). We note that the scenario of the impact event is applied to explain the splitting and outburst events for other comets (Beech 2001, Toth 2001, Gronkowski 2004) but these are only optional and less probable scenarios in the present-day solar system. The intrinsic properties of the asteroid-comet transition objects are the most plausible causes of their unusual behavior. The discovery of the E-P's recurrent activity by Hsieh et al. (2004) in 2002 ruled out the one-time impact event hypothesis as a direct cause of the long-lasting recurrent comet-like activity. But in 2003 they have not found any comet-like activity despite that they used the Keck I 10-m telescope. However, very recently Lowry \& Fitzsimmons (2005) published their 2002 observations of E-P, which were made on 13 July 2002, and they reported very small dust activity with a small dust trail (or tail). They proposed that the evolving of the dust trail started just prior to their observations in July 2002. BVRI observations of E-P obtained by Delahodde et al. (2004) in 2000 are consistent with a dormant (inactive) phase of this object, as no coma or tail (trail) were detected.

Hsieh et al. (2004) suggested various conceptions to explain the observed recurrent comet-like behavior of E-P. The most preferable idea is connected to the comet hypothesis, i.e., the 1996 and 2002 emission events are consequences of seasonal insolation variation, the area of exposed surface volatiles must be located near one of the rotational poles of the nucleus. This volatile region would then only receive enough solar radiation to become active when the near pole tilts toward the Sun, that is that hemisphere's "summer". This like object can be denominated as "activated asteroid" which remains consistent with the possibility of seasonal modulation of E-P's activity (R.P. Binzel, cf. Hsieh et al. 2004). The question is raised whether where E-P exhibited comet-like activity and where it was inactive along its orbit, i.e., how long the periods of activity and dormancy were. For this purpose Toth (2005b) continued the searching for comet-like activity by CCD imaging observations about E-P, which were conducted in January and February of 2005. In addition, mapping of the location of the active and inactive phases of E-P along its orbit (Fig. 4), and taking into account the lightcurve observations which are up to now available in the literature the limitations of the possible rotational pole orientation of this object can be estimated.

In order to find more main belt asteroids which show comet-like activity Hsieh (2004) conducted a deep optical survey of selected main belt asteroids in search of E-P-like objects. Hsieh (2004) presented interim results of this on-going survey. 63 Themis-zone asteroids were selected for this purpose, among others 1999 TU71, but none of them exhibited comet-like activity. Hsieh (2005) (this conference) reviewed the evidence for volatile sublimation as the cause of E-Ps comet-like activity, presented an overview of our ongoing Search for Active Main Belt Asteroids (SAMBA) project that aims to find other E-P-like objects, discussed their progress to date, and considered the implications of this work.

There is another possible explanation, which has not been tested yet for the active asteroids. This mechanism is the removing of the regolithic material from asteroid surface: 


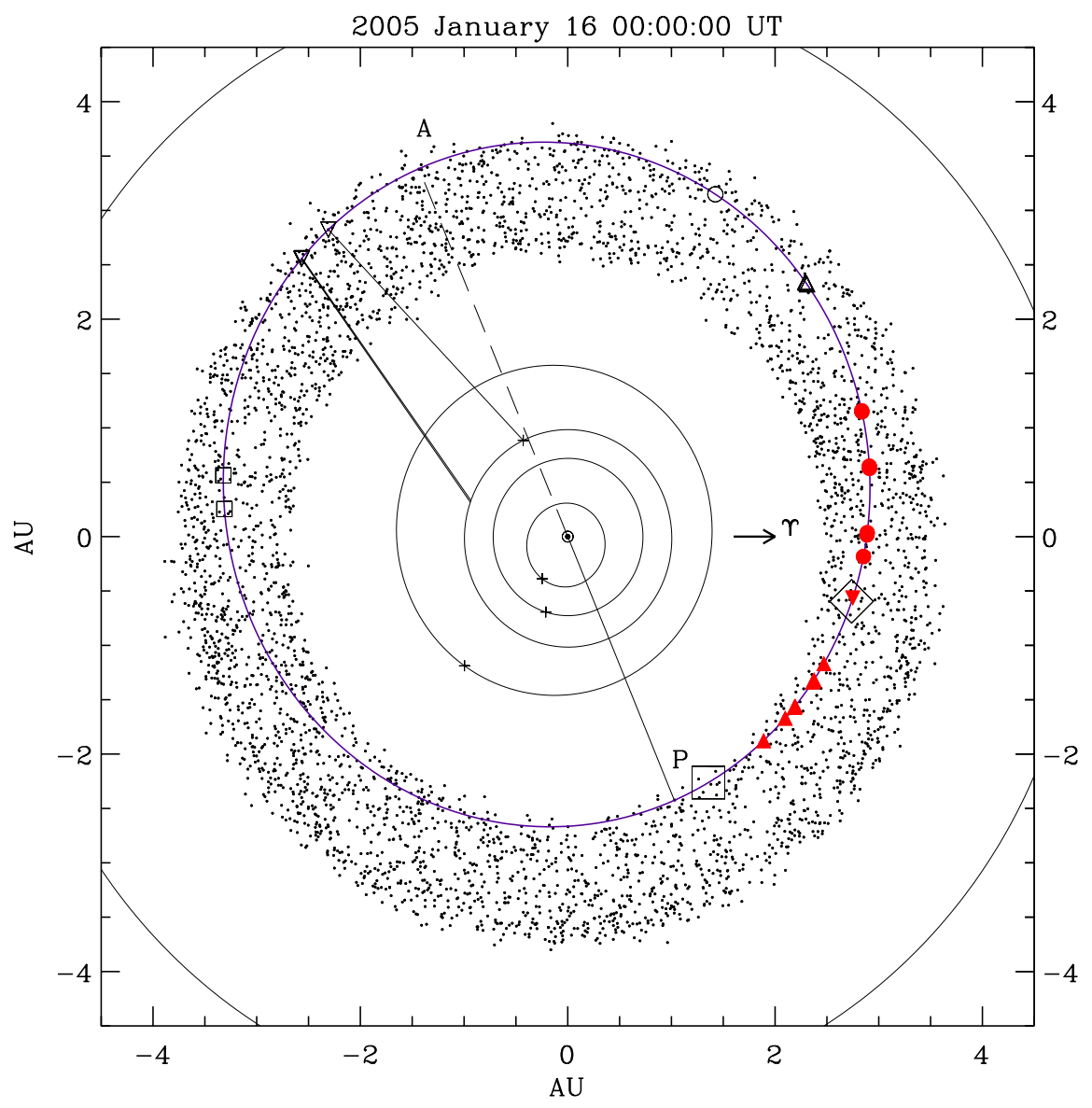

Figure 4. Comparison of the active and dormant phases of the asteroid-comet transition object 7968 Elst-Pizarro (133P/Elst-Pizarro) in its orbit has shown. The orbits and the instantaneous positions of the objects are projected onto the plane of ecliptic for the date of UT $0 \mathrm{~h}$ 16 January 2005. The orbit of 7968 Elst-Pizarro is drawn (solid blue line). Direction of its perihelion point $(\mathrm{P})$ is marked by a solid line connecting the Sun and the point in the orbit. Direction of aphelion (A) is marked by a dashed line. Solid line connects Earth and Elst-Pizarro on the date of the observations taken on UT 16 January, 28 February, and 1 March 2005, respectively. Background asteroids in the Themis-zone are also plotted (small black dots). The positions of the major planets from Mercury to Jupiter are marked with crosses. The orbits of major planets from Mercury to Jupiter are drawn with thin solid lines. Vernal Equinox direction is indicated. Observations with reported dust trail (trail) activity are higlighted with solid red symbols: observations taken in 1996 are solid triangles, observations by Hsieh et al. (2004) from July to December 2002 are solid dots. Observation taken by Lowry \& Fitzsimmons (2005) in July 2002 is shown by a red triangle with downward directed peak, just near to the 1985 observation made by McNaught et al. (1996). Observations which did not report any comet-like activity are represented by open black symbols: big open square (1979), open diamond (1985), open triangles with upward peak (1997), small open squares (2000), open circle (2003), and open triangles with downward peak (2005 observations by Toth 2005b).

it is the effect of solar radiation pressure on small regolith particles Scheeres (2005). The radiation pressure can play important role in migration of surface dust particles, whether or not electromagnetic levitation or seismic shaking is present. We are raising now the question, which is still open, that this mechanism can be effective in case of the E-P. 
However, there are other mechanisms which may work on the surface of active asteroids. Kargel (1991) proposed a specific activity of asteroids and we quote this idea only for the purpose of completeness of a possible explanation for the activity in the asteroid belt, at least to explain the exposition of icy material on the surface. It is the brine volcanism, in which $\mathrm{MgSO}_{4}$ and $\mathrm{Na}_{2} \mathrm{SO}_{4}$ are shown by carbonaceous chondrite mineralogies and elemental abundances to be the most important solutes, is presently suggested to account for the resurfacing (it was observed on some icy satellites). The question is that can this process work in small asteroids or only in larger bodies.

Recent progress for modeling of the mass loss from cometary nucleus may adopt and translate to the activity of asteroids analogously. Quite recently, aside the well known and well elaborated models of gas and dust production of comets, there are other new models, which were discussed for the material releasing activity of cometary nuclei: $(i)$ one of these the model of subsurface "geysers" for the collimated jets, which was proposed by Yelle et al. (2004); (ii) another one is the non-active area based interacted hydrodynamic flow model (see the summaries of the advanced new jet 3-D model by Crifo et al. (2002), and Rodionov et al. (2002)); (iii) surface depression and ice releasing model by Laufer et al. (2005).

Finally, where is the water in the asteroids, which can drive comet-like activity: outgassing, to develop gas coma and to release dust? In general, the question of the occurrence of water ice in primitive asteroids was considered by Barucci et al. (1996). They reviewed the literature dealing with possible signatures of ice in some asteroids (see also various chapters in books Asteroids, Asteroids II, Asteroids III of University of Arizona Press). The subject is poorly developed, even if in the last decade several authors started a spectroscopic survey of asteroids in the visible and near-infrared wavelengths in order to find ice features on asteroid spectra. Aqueous alteration of the mineral assemblages has been claimed for a number of B-, F- and for more than $50 \%$ of the observed C-type asteroids. The compositional and, consequently, the thermal structure of the asteroid belt supports the hypothesis that ices of volatile elements should be more abundant in the external region of the belt, where pristine materials have not undergone drastic modification processes after the accumulation in the planetesimal swarms between those of Mars and of Jupiter. Still there is no evidence of any water, water ice or aqueous alteration materials on the D-type asteroids, which are considered the least altered objects.

The Dawn mission addresses the long-standing goals of understanding a "wet" relict of the formation of the solar system: it is the 1 Ceres (see Mousis \& Alibert 2005, this conference). From the long-exposure IUE spectra A'Hearn \& Feldman (1992) already detected $\mathrm{OH}$ in the northern limb of Ceres. They concluded that amount of $\mathrm{OH}$ is consistent with a polar cap that might be replenished during winter by subsurface percolation, but which dissipates in summer. Under the assumption that most of the volatiles were not vaporized during the accretion phase and thermal evolution of Ceres, utilizing a timedependent solar-nebula model, Mousis et al. (2005) determined tbe volatile abundances $\left(\mathrm{CO}_{2}, \mathrm{CO}, \mathrm{CH}_{4}, \mathrm{~N}_{4}, \mathrm{NH}_{3}, \mathrm{Ar}, \mathrm{Xe}, \mathrm{Kr}\right.$ with respect to the water). Recently, high signal to noise spectra of Ceres were obtained by Vernazza et al. (2005) with the NASA IRTF. They confirmed the presence of the 3.06 micron absorption feature. Laboratory measurement of ion-irradiated organics and ices suggest that this feature can be reproduced with a linear mixture of crystalline ice and residues of ion-irradiated asphaltite.

Campins \& Lauretta (2004) predicted that a significant fraction of cometary solids could be in the form of hydrated silicates. For example, in comet Hale-Bopp the observed spectral features attributed to anhydrous crystalline silicates can be produced by as little as $15 \%$ of the particles; the rest of the silicates could be a mixture of hydrated and amorphous grains. This is in part because anhydrous crystalline silicates are easier to 
identify spectrally than hydrated or amorphous silicates. Campins \& Lauretta (2004) quoted the model of chondrule-forming shock waves in icy regions of the early solar nebula, which can produce rapid mineral hydration, thus supporting a nebular origin for the hydrated silicates in the chondrule rims. Previous arguments had suggested hydration had to occur in parent bodies and not in the nebula. Hence, comets may have also accreted hydrated silicates from the solar nebula. Observationally, cometary hydrated silicates could be detected using near-infrared reflectance spectroscopy of cometary surfaces and mid-infrared spectroscopy of cometary dust. There is an ongoing spectroscopic survey in the visible and near-infrared to observe the surface of cometary nuclei and related minor bodies by Licandro et al. (2002).

\section{Connections between asteroids and comets in the Near-Earth space: recent progress}

Asteroids and comets supply the meteoritic material and dust in the interplanetary space (see reviews by Mann et al. 2004, Mann 2005, and the relevant chapters of the Asteroids III 2002 and Comets II 2004 books, University of Arizona Press). In the NearEarth region the most abundant source of the interplanetary dust and meteoroids is the Taurid-Encke complex, which contains both asteroids, comets and meteor streams (Asher \& Clube 1997, see many chapters in the book Hazards due to comets and asteroids. Univ. of Arizona Press, Tucson, 1995). Resonances and chaotic motions are important mechanisms to deliver both the asteroids and comets into the inner-Earth regions even sungrazing orbits. So, in the Near-Earth and inner-Earth regions both the comets and asteroids are the abundant sources of the interplanetary dust and meteoritic material but the relative contribution of these sources are not quantified (Biesecker et al. 2002, Mann et al. 2004). However, based on an ongoing optical/thermal infrared photometric imaging survey of the dust mass loss from comets Lisse (2002) demonstrated that there is an evolution with time of the kind of dust emitted from a cometary nucleus surface. His results indicate that the mass loss rate from the ecliptic comets alone is enough to supply the interplanetary dust complex against losses. Moreover, based on the ISO and MSX (Midcourse Space Experiment) infrared data Lisse et al. (2004) concluded that if the dust emission behavior of $2 \mathrm{P} /$ Encke is typical of other ECs, then comets are the major suppliers of the interplanetary dust cloud. Regarding the nucleus of $2 \mathrm{P}$, during its 2003 apparition high resolution spectroscopic observations made using the TNG telescope on La Palma by Saba et al. (2004). Spectrum of 2P is poor of spectral features: only a few emission lines were found $\left(\mathrm{C}_{2}, \mathrm{NH}_{2}, \mathrm{H}_{2} \mathrm{O}^{+}\right)$as like in ECs but this may indicate low gas production and it is in agreement with previous observations of $2 \mathrm{P}$ pointing at a dark and dusty object.

Fernández et al. (2002), and Pittich et al. (2004) calculated that Jupiter-family comets can reach the Near-Earth region. Pittich et al. (2004) modeled the transfer routes from Jupiter-family towards Encke-like cometary orbits. They found that resonances and nongravitational forces appear to be key factors in the transfer of orbits.

Recent progress in the studies of physical properties of the Near-Earth Objects in connection with comets done by Fernández et al. (2005) who presented the results of a mid-infrared survey of 26 asteroids in comet-like orbits, including six Near-Earth asteroids (NEAs). Merging this with their earlier study of low albedo objects (Fernández et al. 2001) they analized 32 objects. They defined a "comet-like" orbit as one having a Tisserand invariant $T_{J}$ under 3 (but only including objects that are NEAs or otherwise unusual). Visible-wavelength data were also obtained, so geometric albedos (in the Cousins $R$ band) and effective radii are presented as derived using the NEA Thermal 
Model. Nine of their objects were observed at two or more mid-infrared wavelengths, and in all cases the low-thermal inertia thermal model was found to be applicable, with various values of the beaming parameter. Their work more than quintuples the total number of observationally constrained albedos among $T_{J}<3$ asteroids to 32 . Defining the "comet-like" albedos as those below 0.075 , they found that $64 \% \pm 5 \%$ of the sample has comet-like albedos. Objects in comet-like orbits with comet-like albedos are candidates for being dormant or extinct comets. They found a very strong correlation between the albedo distribution and $T_{J}$, with the percentage of dark $T_{J}<3$ asteroids being much greater than that of the $T_{J}>3$ NEAs. There are 10 NEAs among the 32 objects, and of those, $53 \% \pm 9 \%$ have comet-like albedos. With the current crop of NEAs, this implies that about $4 \%$ of all known NEAs are extinct comets. A comparison of the histogram of $T_{J}<3$ asteroid albedos with that of active cometary nuclei shows that the former has a larger spread.

Hsieh \& Jewitt (2005) presented deep optical imaging of Geminid meteor stream parent Apollo asteroid 3200 Phaethon taken in search of low-level cometary activity (i.e., coma or dust trail). Although no unambiguous cometary behavior was observed, they found an upper limit on the object's cometary mass-loss rate of $M_{\lim } \sim 0.01 \mathrm{~kg} \mathrm{~s}^{-1}$. The corresponding active fraction (the fraction of the surface area that could consist of freely sublimating water ice) is $f \leqslant 7 \times 10^{-6}$, at least 2 orders of magnitude smaller than other known comets.

\section{Current status and outstanding questions in connections between asteroids and cometary nuclei}

However, there are outstanding questions of the connections between asteroids and cometary nuclei, even the physical characteristics of these objects like size determination, size distribution, surface and bulk interior properties, as well as the question of binary objects among the asteroids and cometary nuclei. The current status and outstanding questions are summarized as follows.

\subsection{Sizes and shapes}

Remarkable progress has been made during the past decade in measuring the sizes of cometary nuclei and related primitive small bodies of the solar system (TNOs, Centaurs, and primitive asteroids) but it is also clear that this field is still in its infancy. Weissman et al. (2002), Lowry \& Weissman (2003), Barucci et al. (2004), Jewitt (2004), and Lamy et al. (2004) reviewed the recent status of the sizes and shapes of small bodies in the solar system, including comets and related asteroids. The current best estimate, $q_{S}=1.9 \pm 0.3$ for ECs, is conspicuously different from that of the KBO and Centaur populations, but is similar to that of the NEOs (Lamy et al. 2004). This value also corresponds to that of a collisionally evolved population with pure gravity scaling, but we re-emphasize that O'Brien \& Greenberg (2003) showed that this distribution is, in fact, wavy in the size range relevant to ECs. In addition, Pan \& Sari (2005) refined the modeling approximation for the size distribution of the KBOs, which has to be tested for larger observational data samples.

A totally open issue is the nature of the size distribution of cometary nuclei at the small end of the spectrum. Does the relatively steep power law derived from the intermediatesized objects extend indefinitely to smaller sizes? Or is the size distribution truncated at some value that depends on the physical formation mechanism (e.g., gravitational instability within the solar nebula) or destruction mechanism (e.g., total disruption)? 
What is the bias in ecliptic comet discoveries and how does that affect the current distribution of sizes? Why do we observe so few large $\left(r_{n} \gtrsim 5 \mathrm{~km}\right)$ cometary nuclei?

What are the correct size distributions of NICs and Damocloids and how does they relate to that of Damocloids, and what can be the result of the comparison of their other physical properties?

How does evolution affect the physical properties of cometary nuclei? Is there really a continuum of surface properties that is dependent on the activity level and physical evolution (e.g., with a youthful Chiron at one end and an aged $2 \mathrm{P} /$ Encke and extinct comets with asteroidal appearance on the other)?

Splitting events (Boehnhardt 2004) obviously affect the size and shape of small bodies (Jewitt 2004), and how do we estimate their effect on the distribution functions? Perhaps better data on the splitting rates of comet nuclei, coupled with a better understanding of the physical mechanism(s) for splitting events, will help to resolve these issues, but that remains to be seen.

\subsection{Surface properties}

Comparing the size and albedo determinations, the situation for albedos of comet nuclei and related asteroids is even worse, in the sense that reliable values are available for only about a dozen objects. Nevertheless, we are struck by the relatively small range in the albedo $(0.04 \pm 0.02)$, which suggests that the surfaces of cometary nuclei are exceptionally dark, contrary to the early expectations for these "icy" bodies.

The phase function of atmosphereless bodies, both cometary nuclei and primitive asteroids, offers a powerful means for investigating the properties of surface (e.g., roughness and single-particle albedo). Observations of the detailed phase function of the inactive nucleus of comet 28P/Neujmin 1 by Delahodde et al. (2001) yielded important results (cf. reviews by Campins et al. 2001, Campins et al. 2003). They found that the average colours of the nucleus are similar with those of D-type asteroids and other comet nuclei. The phase function obtained for the nucleus has a linear slope of $0.025 \pm 0.006 \mathrm{mag}$ $\mathrm{deg}^{-1}$, less steep than that of the mean C-type asteroids. At smaller phase angles, the function steepens and a strong opposition effect appears at $\alpha \lesssim 1.5^{\circ}$. However, Lamy et al. (2004) noted that this effect, comparable to those found on medium geometric albedo $(\sim 0.15)$ M-type asteroids, and icy satellites, is quite surprising for a cometary nuclei. As surface ice is excluded on such a low activity nucleus, a high surface porosity could perhaps be invoked, but this possible interpretation has not been investigated. The first results of Licandro et al. (2002) showed that the spectrum of the bare nucleus of comet $28 \mathrm{P} /$ Neujmin 1, which is the less active in the Jupiter-family, exhibited rotational variation: the spectral change appears in the infrared spectral slope and it is consistent with sporadic changes in the $V, R$, and $I$ bands reported in this comet by Delahodde et al. (2001). Moreover, there is another very recent example which supports the connections between dark C-type asteroids and cometary nuclei. Combining the disk integrated magnitudes calculated from the Deep Space 1 images with the HST and ground-based measurements Soderblom et al. (2002) and Buratti et al. (2004) determined the phase function of the nucleus of $19 \mathrm{P} /$ Borrelly over a large range of phase angle, from $3^{\circ}$ to $88^{\circ}$. The phase curve is very similar to that of the dark C-type asteroid 253 Mathilde.

It is also difficult to obtain reliable color data on cometary nuclei. While the color of the nucleus itself does not provide unique information on the physical properties, color data are useful for comet-to-comet comparisons, which may suggest differences in surface properties, and, especially, in making comparisons with other minor bodies in the solar system (e.g., Centaurs, TNOs, and asteroids). The colors of cometary nuclei are diverse, 
with some being highly reddened compared to solar color, some being neutral, and a few having a slightly blue color.

An important, unresolved issue concerns the interpretation of disk-integrated thermal measurements, which, in principle, provide robust determinations of sizes and albedos. The so-called Standard Thermal Model for asteroids is often used to interpret cometary thermal data, although its applicability to objects having a mixture of dust and ice is questionable (see examples of the model in Groussin et al. 2004, and review of the thermal infrared models and their application and limitations Lamy et al. 2004).

Does the P- and D-type asteroids have organic or silicate surface, or in other words: do we face with a new paradigm? A recent extensive spectroscopic survey of asteroids by Emery et al. (2005) in order to study the mineralogy of asteroids from observations with the Spitzer Space Telescope led to a very important implication for the D-type asteroids including Jupiter Trojans among which there are many D-class objects. They suggested a silicate nature for the surface of Trojan asteroids based on the absence of organic absorption in 3- to 4 micron region and on the ability to model the vis-NIR spectra with silicates alone. These new results from Spitzer/IRS support a silicate interpretation. This challenges the old paradigm. i.e., the view dominant since the early 1980's that the low albedo and red spectral slopes of P- and D-type asteroids are due to organics on the surfaces. The middle part of the early solar nebula may not have been as rich in organic material as once thought. Emery et al. (2005) also found similar spectrum for a few Centaurs and one of them shows a very similar spectrum to 624 hektor. This can be a new paradigm for P- or D-type asteroids, including the Jupiter Trojans and one part of the Centaur population. However, either the observed or composite phase function of an organic material covered surface and the only silicate material covered surface should be different. The observed phase function of the dark primitive asteroids do not support the only silicate surface material (cf. Belskaya et al. 2003)). In addition, the analyses of the Tagish Lake meteorite, which is considered that originated from a P- or D-type asteroid, showed that it is similar to the hydrous minerals (Hiroi et al. 2003), and the surface regolith of these asteroids may be made of intermediate materials of the $\mathrm{CI} / \mathrm{CM}$ chondrites (Hiroi et al. 2004).

Regarding the activity of the asteroid-comet transition objects, the recurrent cometlike activity of $133 \mathrm{P} /$ Elst-Pizarro still waits for the convincing explanation (see the details and model scenarions in Section 8). Perhaps, the asteroid flyby target of Comet Odyssey mission, the Themis-zone object Elst-Pizarro will be selected.

\subsection{Bulk interior properties}

Among the key scientific objectives of the Deep Impact project there are questions which are connected to the comet-asteroid interrelations. Namely, $(i)$ comets have the most primitive, accessible material in the solar system, but we do not know what is hidden below the evolved subsurface layers (material composition, interior structure, bulk density and porosity, etc.), (ii) there must be many dormant comets masquerading as asteroids but the questions is that we do not know how to identify these bodies, and (iii) comets must become dormant but we do not know whether ice is exhausted of sublimation is inhibited.

Although cometary nuclei generally contain much more ice than asteroids do, perhaps the "evolved" comets share many common characteristics with asteroids. In addition, at least some asteroids, and many cometary nuclei, are thought to have porous, "rubblepile" physical structures (Davis et al. 1985, Weissman 1986). Does this point to commonalities in their formation mechanism? There are various model conceptions on the internal structure of comets (Weissman et al. 2004) but we have not verified these yet. 
Weidenschilling (2004) reviewed the process of the formation of cometesimals as collisionally processed aggregates, i.e., rubble piles (see also Weissman et al. 2004). Although his 1-dimensional models suggest preferential formation of bodies having a characteristic size of $\sim 100 \mathrm{~m}$, his more recent 2-dimensional models have a size distribution that is more closely approximated by a power law with only a small depression centered near $1 \mathrm{~m}$ in size. The observed fragments of $73 \mathrm{P} /$ Schwassmann-Wachmann 3 (Toth et al. 2005) and the numerous small, unobserved fragments whose existence is suggested by the results presented on this split comet, may be consistent with Weidenschilling's model, but the observations cannot be used to confirm that model in detail. However, the observed fragments of C/1999 S4 (LINEAR) (Weaver et al. 2001), which is a NIC, may confirm this model.

The Stardust images of $81 \mathrm{P} /$ Wild 2 raised the question that the cometary nuclei can be diverse in their internal structure and not only in their surface properties due to evolutionary processes (aging, erosion, volatile depletion, etc.), even the nucleus of $81 \mathrm{P} /$ Wild 2 is not a rubble pile (Weaver 2004). According to the Stardust images It was suggested that the nucleus of $81 \mathrm{P}$ held together by internal strength rather than by gravity alone. But Asphaug et al. (2002) concluded that instead of competing for dominance, strength and gravity collaborate over a wide spectrum of sizes and shapes to produce some of the richest structure in nature. Both 19P/Borrelly (Deep Space 1), 81P/Wild 2 (Stardust images), and 9P/Tempel 1 (Deep Impact) are ecliptic comets but there have different surface and possibly interior structures. 1P/Halley (Vega 1 and 2, Giotto) images of this NIC also show differences from the nuclei of these ecliptic comets. Are there different groups of comets in internal and surface properties, i.e., are there different groups among the ECs, and are the EC nuclei different from those of NICs including HTCs or Damoclods?

Study of the stability against rotational disintegration of the minor bodies allows to constrain their bulk interior properties and possibly their evolution and interrelations between them. This old approach is renewed Toth \& Lisse (2005) applying the new models developed by Davidsson 1999. Toth \& Lisse (2005) estimated the regions of stability, fragmentation, and destruction for cometary bodies versus rotational breakup in the radius - rotational period plane. They have applied the model of Davidsson (1999) for rotational stability of spherical bodies to study the location of observed comets and cometary asteroids in the radius - rotational period plane. In doing so, they considered the range of plausible cometary nucleus material strengths and bulk densities as found in the literature (Fig. 5). We emphasize here that the spherical, solid, homogeneous, rigid, and incompressible body, which is the starting-point of most physical models, can too easily be oversimplified (Davidsson 1999). While it would be interesting to directly model a cometary nucleus with more complex models, there are no direct measurements of the internal material properties of cometary nuclei and related objects.

Toth \& Lisse (2005) found that the boundaries of rotational stability move significantly in the $\left(R, P_{\text {rot }}\right)$ plane as the material properties change. However, the location on the the rotational period - body radius plane, with respect to the rotational breakup limit curves, still allows us to estimate the possible numerical values for the internal strength and bulk density using a statistical approach. E.g., the existence of $95 \mathrm{P} /$ Chiron as a coherent body can be accommodated only if its material strength is characteristic of relatively strongly structured material - e.g., stronger than the weak nuclei advocated by Greenberg et al. (1995). Moreover, the bulk density constraint for a shear fractured body yields a somewhat larger mean bulk density of Chiron than the bulk density of an aggregate structured cometary nucleus. The large majority of observed comets lie in the allowed region for all possible nuclear constituent properties (Fig. 6). They suggest a 

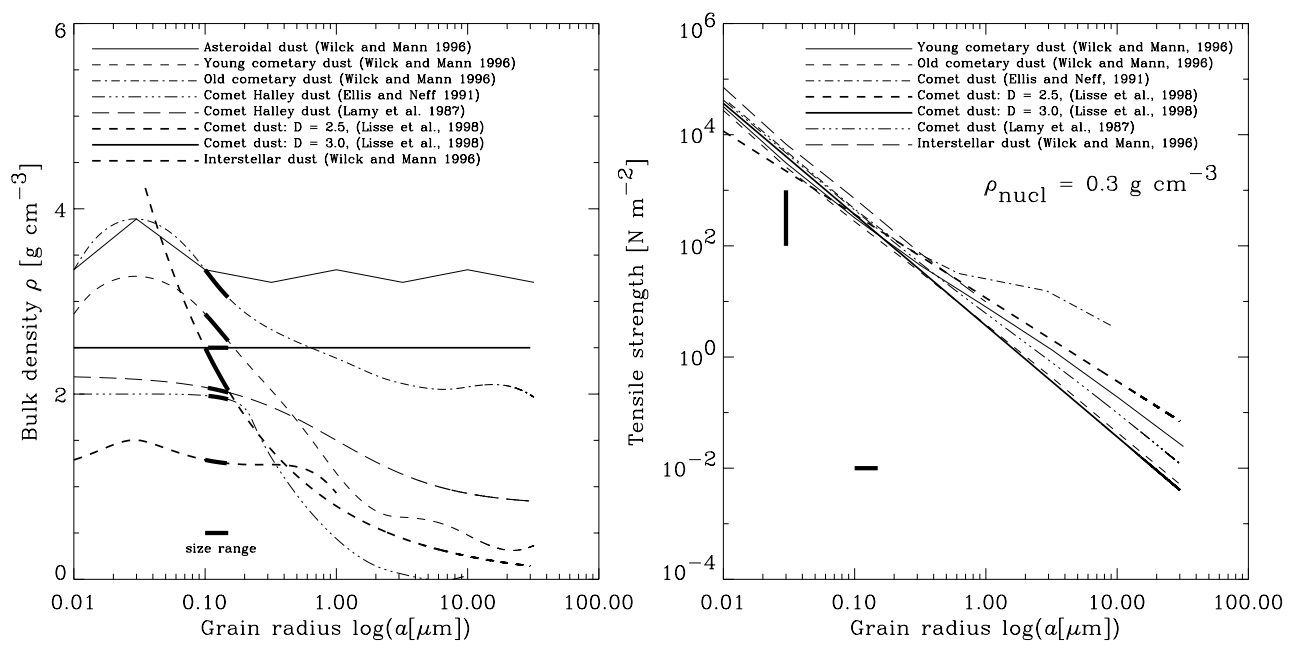

Figure 5. Left panel: Size dependence of the bulk density of spherical dust grains as given in the literature (Toth \& Lisse 2005 and references therein). The bulk density law is computed for two fractal dimensions: $D=2.5$ (porous dust grains) and $D=3$ (solid grains). The size range of ISM dust particles $(0.10-0.15 \mu \mathrm{m})$ which are presumed to be the basic building blocks of the cometary nucleus is marked by a horizontal bar. The bulk density range of the dust grains is indicated by thick solid parts in the curves. Right panel: Tensile strength for aggregate dust grain types as given by Wilck \& Mann (1996) and as calculated for the dust grain bulk density functions given in the litarature. The bulk density law is computed for two fractal dimensions: $D=2.5$ (porous dust grains) and $D=3$ (solid grains). The size range $(0.10-0.15 \mu \mathrm{m})$ of dust particles which are presumed to be the basic components of the cometary nucleus is marked by the horizontal bar. The typical tensile strength range is indicated by the vertical bar. The assumed bulk density of the nucleus is $\rho_{\text {nucl }}=0.3 \mathrm{~g} \mathrm{~cm}^{-3}$. Figures from Toth \& Lisse (2005).

correlation between the proximity to the rotational breakup instability and the surface inhomogenities or activity for the primitive minor bodies.

\subsection{Binary asteroids and satellites of cometary nuclei}

While the occurrence of satellites for both main belt asteroids, Near-Earth asteroids, Transneptunian objects, and Trojan asteroids is steadily growing, there is still no definite, observational evidence that binary cometary nuclei exist. Asteroids do have satellites, and the properties of the known asteroidal binary systems were reviewed by Merline et al. (2002). Very recently the problem of the existence of satellites of cometary nuclei was discussed by Lamy et al. (2004). The detection of a satellite companion to a cometary nucleus would be of unique value as it would provide access to the mass of primary. If the mass of the nucleus is known, and if the size is independently derived, then the mean bulk density and porosity can be calculated, providing insight into the internal properties of the nucleus. However, a binary system must have long-term orbital stability and the components must be large enough ("bodies"), i.e., we do not consider for example the submeteoroid sized debris as a component. But may the binary cometary nuclei exist - or if they do not exist, why? Intuitively, one possible explanation is the rapid divergence of the fragments of split comets due to the strong nongravitational forces (cf. the calculations for the fragments of C/1996 B2 (Hyakutake) by Desvoivres \& Levasseur-Regourd 2002 and references therein).

We draw attention that still there is no any binary Centaur observed. The number of known binary TNOs is growing but still there is no known binary among the Centaurs. If 

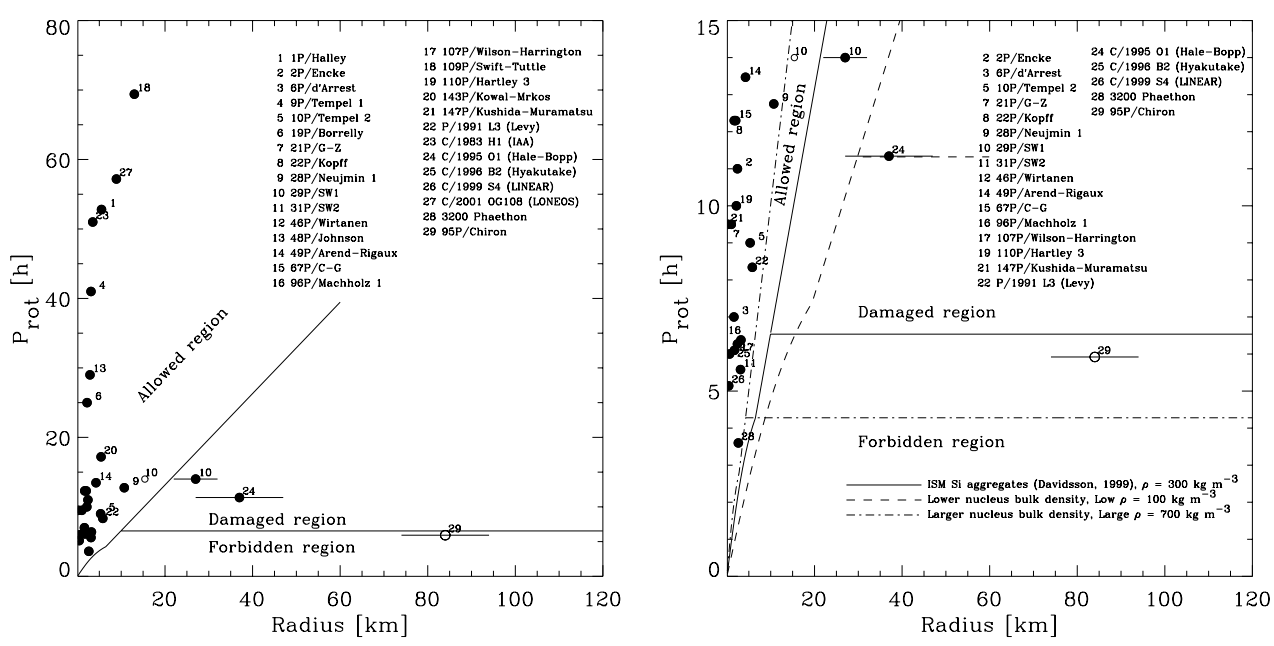

Figure 6. Left panel: The critical rotational breakup limits for spherical small bodies assuming a bulk density of $\rho=300 \mathrm{~kg} \mathrm{~m}^{-3}$ and material strength according to Greenberg et al. (1995). The calculated limits were derived using the model of Davidsson (1999). Measured cometary rotational periods and radii for all the cometary objects in the well-observed dataset are represented by filled circles. The location of the nucleus of disrupted comet C/1999 S4 (LINEAR), before breakup, is also shown (Object No. 26). The two effective radii of 29P/Schwassmann-Wachmann 1 (object No. 4) are reported assuming that the rotational period is the same. The active Centaur 95P/Chiron is shown by a circle (object No. 29). Right panel: Same as for the left panel, except the vertical axis has been expanded to show the detail of the higher rotation rate objects. Border lines for lower and larger bulk densities of the nucleus are also plotted. Figures from Toth \& Lisse (2005).

the Centaurs come from TNOs, among which the duplicity is common, therefore binary Centaurs should exist. The Centaurs are the possible progenitors of ECs therefore the mass and bulk density of binary Centaurs are important and these can be be determined in binary systems. Toth (1999) has already studied the orbital stability and detectability of possible Centaur binaries.

A possible byproduct of the successful Deep Impact experiment could be the discovery of a possible satellite formation from the reaccreted ejected cometary material but the probability of this process is extremely unlikely comparing to the satellite formation from reaccreted material which is an effective process in asteroids as it was described by Michel et al. (2001). However, the problem of the satellites to cometary nuclei should be explored via modeling and observations in the future.

\section{Concluding remarks}

When David Jewitt (Jewitt 1992) discussed the physical characteristics of cometary nuclei and related asteroids in the context of current models of their origin and evolution, and Jane Luu presented the first modern review of the connection between cometary nuclei and related asteroids (Luu 1994) on the basis of physical properties of these objects, more than 10 years ago, their data samples were limited to a few comets, the 2 Centaurs (Chiron and Pholus), a few Jupiter Trojans and other asteroids for which physical measurements were available. Review by Luu (1994) incorporated comparison between the orbital characteristics (groups of minor bodies) and surface properties. At the 1996 Asteroids, Comets, and Meteor (ACM 1996) conference, Karen Meech's review discussed 
17 ECs, 7 Halley-type comets (HTC sub-group of the NICs), and 2 Centaurs (Chiron and $29 \mathrm{P}$ ) in relation with their physical characteristics, even discussing their bulk interior physical properties. At the 2002 Asteroids, Comets, and Meteor (ACM 2002) conference, David Jewitt summarized the results of the origin and evolution of comets including the ecliptic comets and related primitive asteroids according to the data from growing observational samples available as of 2002. For the Asteroids III book (2002), the discussion of the interrelations between comets and related asteroids (TNOs, Centaurs, Jupiter Trojans, and other primitive asteroids) based on large samples of the observations, detailed physico-chemical models, and celestial mechanical studies (see reviews by Barucci et al. 2002, Marzari et al. 2002, Weissman et al. 2002). For the Comets II book (2004), the study of cometary nuclei is divided among 10 chapters, with questions such as the physical properties, rotation, surface properties, internal properties, and the relationship with other minor bodies deserving their own, separate chapters (see reviews by Barucci et al. 2004, Jewitt 2004, Lamy et al. 2004, Weissman et al. 2004) We are far from fully understanding the cometary nuclei and asteroid-comet transition objects (e.g., $133 \mathrm{P} /$ Elst-Pizarro), as illustrated above by the list of outstanding issues. On the other hand, the future is bright as new facilities will soon allow us to push the present limits of our observational capabilities even further. Both the large ground-based telescopes (e.g., Keck, Gemini, Very Large Telescope, Pan-STARRS. Large Synoptic Survey Telescope (LSST), ALMA) and infrared space telescopes (Spitzer, Herschel) will provide detection of faint small bodies, measuring the sizes, shapes, albedos, colors, rotational parameters, thermal characteristics. Meanwhile forthcoming cometary space missions (e.g., Stardust sample return in 2006, Comet Odyssey, Rosetta), and some asteroid related missions (Dawn and Gulliver) will provide new results in the asteroid-comet connections. The Rosetta lander will perform a broad range of in-situ observations and analysis of the surface and subsurface regions of the nucleus. These will allow us to study a few cometary nuclei and related asteroids in unprecedented detail.

\section{Acknowledgements}

Author acknowledges the beneficial cooperation with Philippe Lamy, as well as Michael A'Hearn, Laurent Jorda, Horst Uwe Keller, Zdenek Sekanina, Nicholas Thomas, Harold Weaver for the collaborations using the Hubble Space Telescope for many years. I. T. thanks Paul Abell, Yanga Fernández, Henry Hsieh, David Jewitt, and Paul Weissman for the discussions on the asteroid-comet transition objects, Michael Belton, Björn Davidsson, and Casey Lisse for the discussions on the bulk interior properties of cometary nuclei, as well as This work was partly supported by the French "Programme National de Planétologie", jointly funded by CNRS and CNES, and of the bilateral French-Hungarian cooperation program. I. T. acknowledges the support of the Université de Provence, of the Hungarian Academy of Sciences through grant No. 9871.

\section{References}

Abell, P.A., Fernández, Y.R., Pravec, P., French, L.M., Farnham, T.L., Gaffey, M.J., Hardersen, P.S., Kusnirak, P., Sarounova, L., \& Sheppard, S.S. 2003, LPSC 28, Abstr. No. 1253 A'Hearn, M.F. \& Feldman, P.D. 1992, Icarus 98, 54 A'Hearn, M.F., Millis, R.L., Schleicher, D.G., Osip, D.J., \& Birch, P.V. 1995, Icarus 118, 223 Asher, D.J., Bailey, M.E., Hahn, G., \& Steel, D.I. 1994, Mon. Not. R. Astr. Soc. 267, 26 Asher, D.J. \& Clube, S.V.M. 1997. Cel. Mech. Dyn. Astr. 69, 149 Asphaug, E., Ryan, E.V., \& Zuber, M.T. 2002, in: W.F. Bottke, A. Cellino, P. Paolicchi \& R.P. Binzel (eds.), Asteroids III, (Tucson, Arizona: The University of Arizona Press), 463

Barucci, M.A., Fulchignoni, M., \& Lazzarin, M. 1996, Planet. Space Sci. 44, 1047 
Barucci, M.A., Cruikshank, D.P., Mottola, S., \& Lazzarin, M. 2002, in: W.F. Bottke, A. Cellino, P. Paolicchi \& R.P. Binzel (eds.), Asteroids III, (Tucson, Arizona: The University of Arizona Press), 273

Barucci, M.A., Doressoundiram, A., \& Cruikshank, D.P. 2004, in: M.C. Festou, H.U. Keller \& H.A. Weaver (eds.), Comets II, (Tucson, Arizona: The University of Arizona Press)

Bauer, J.M., Fernández, Y.R., \& Meech, K.J. 2003, Publ. Astr. Soc. Pacific 115, 981

Beaugé, C. \& Roig, F. 2001, Icarus 153, 391

Beech, M. 2001, Mon. Not. R. Astr. Soc. 327, 1201

Belskaya, I.N., Barucci, A., \& Shkuratov, Y.G. 2003, Earth, Moon, and Planets 92, 201

Bendjoya, P., di Martino, M., \& Saba, L. 2004, Icarus 168, 374

Bernstein, G.M., Trilling, D.E., Allen, R.L., Brown, M.E., Holman, M., \& Malhotra, R. 2004, Astron. J. 128, 1364

Biesecker, D.A., Lamy, P., St. Cyr, O.C., Llebaria, A., \& Howard, R.A. 2002, Icarus 157, 323

Bockelée-Morvan, D., Lellouch, E., Biver, N., Paubert, G., Bauer, J., Colom, P., \& Lis, D.C. 2001, Astron. Astrophys. 377, 343

Boehnhardt, H., Sekanina, Z., Fiedler, A., Rauer, H., Schulz, R., \& Tozzi, G.P. 1998, Highlights in Astron. 11A, 233

Boehnhardt, H., Barucci, M.A., Delsanti, A., De Bergh, C., Doressoundiram, A., Romon, J., Dotto, E., Tozzi, G.P., Lazzarin, M., Fornasier, S., Peixinho, N., Hainaut, O., Davies, J., Rousselot, P., Barrera, L., Birkle, K., Meech, K., Ortiz, J.L., Sekiguchi, T., Watanabe, J.-I., Thomas, N., \& West, R. 2003, Earth, Moon, and Planets 92, 145

Boehnhardt, H. 2004, in: M. Festou, H.U. Keller \& H.A. Weaver (eds.), Comets II, (Tucson, Arizona: The University of Arizona Press)

Bottke, W.F., Morbidelli, A., Jedicke, R., Petit, J.-M., Levison, H.F., Michel, P., \& Metcalfe, T.S. 2002, Icarus 156,399

Brandt, J.C., A'Hearn, M.F., Randall, C.E., Schleicher, D.G., Shoemaker, E.M., \& Stewart, A.I.F. 1998, ASP Conf. Ser. 107, 289

Brown, W.R. \& Luu, J.X. 1998, Icarus 135, 415

Brown, M.E. \& Koresko, C.C. 1998, Astrophys. J. 505, L65

Brown, R.H., Cruikshank, D.P., \& Pendleton, Y. 1999, Astrophys. J. 519, L101

Buratti, B.J., Hicks, M.D., Soderblom, L.A., Britt, D., Oberst, J., \& Hillier, J.K. 2004, Icarus 167,16

Campins, H., Licandro, J., Chamberlain, M., \& Brown, R.H. 2001, Bull. Amer. Astr. Soc. 33, Abstr. [41.08], 1094

Campins, H. \& Fernández, Y.R. 2002, Earth, Moon, and Planets 89, 117

Campins, H., Licandro, J., Guerra, J., Chamberlain, M., \& Pantin, E. 2003, Bull. Amer. Astr. Soc. 35, Abstr. [47.02]

Campins, H. \& Lauretta, D.S. 2004, Bull. Amer. Astr. Soc. 36, Abstr. [21.08]

Chapman, C.R., Merline, W.J., Bierman, B., Keller, J., \& Brooks, S. 1997, Bull. Amer. Astr. Soc. 29, Abstr. [12.10]

Chodas, P.W. \& Yeomans, D.K. 1995, in: K.S. Noll, H.A. Weaver \& P.D. Feldman (eds.), The Collision of Comet Shoemaker-Levy 9 and Jupiter, (New York: Cambridge University Press), 1

Choi, Y.J., Brosch, N., \& Prialnik, D. 2003, Icarus 165, 101

Coradini, A., Capaccioni, F., Capria, M.T., de Sanctis, M.C., Espinasse, S., Orosei, R., Salomone, M., \& Federico, C. 1997, Icarus 129, 337

Crifo, J.-F., Rodionov, A.V., Szegő, K., \& Fulle, M., 2002, Earth, Moon, and Planets 90, 227

Cruikshank, D.P., Dalle Ore, C.M., Roush, T.L., Geballe, T.R., Owen, T.C., de Bergh, C., Cash, M.D., \& Hartmann, W.K. 2001, Icarus 153, 348

Davidsson, B.J.R. 1999, Icarus 142, 525

Davidsson, B.J.R. 2001, Icarus 148, 375

Davis, D.R., Chapman, C.R., Weidenschilling, S.J., \& Greenberg, R. 1985, Icarus 62, 30

Davies, J.K., McBride, N., Green, S.F., Mottola, S., Carsenty, U., Basran, D., Hudson, L.A., \& Foster, M.J. 1998, Icarus 132, 418 
de Bergh, C., Boehnhardt, H., Barucci, M.A., Lazzarin, M., Fornasier, S., Romon-Martin, J., Tozzi, G.P., Doressoundiram, A., \& Dotto, E. 2004, Astron. Astrophys. 416, 791

Delahodde, C.E., Hainaut, O.R., Boehnhardt, H., Dotto, E., Barucci, M.A., West, R.M., \& Meech, K.J. 2000, in: A. Fitzsimmons, D. Jewitt \& R.K. West (eds.), Minor Bodies in the Outer Solar System, (Berlin: Springer-Verlag), 61

Delahodde, C.E., Meech, K.J., Hainaut, O.R., \& Dotto, E. 2001, Astron. Astrophys. 376, 672

Delahodde, C.E., Hainaut, O.R., Dotto, E., \& Campins, H. 2004, Bull. Amer. Astr. Soc. 36, Abstr. [34.05]

Desvoivres, E. \& Levasseur-Regourd, A.C. 2002, in: B. Warmbein (ed.), Asteroids, Comets, Meteors - ACM 2002, (Noordwijk, The Netherlands: ESA Publications Division), ESA $S P-500,645$

Dohnanyi, J.S. 1969, J. Geophys. Res. 74, 2431

Dotto, E., Barucci, M.A., Leyrat, C., Romon, J., de Bergh, C., \& Licandro, J. 2003, Icarus 164, 122

Dumas, C., Owen, T., \& Barucci, M. 1998, Icarus 133, 221

Duncan, M. \& Levison, H.F. 1997, Science 276, 1670

Emery, J.P. \& Brown, R.H. 2003, Icarus 164, 104

Emery, J.P. \& Brown, R.H. 2004, Icarus 170, 131

Emery, J.P., Cruikshank, D.P., Van Cleve, J., \& Stansberry, J.A. 2005, LPSC 36, Abstr. 2072

Farinella, P. \& Davis, D.R. 1996, Science 273, 938

Fernández, J.A., Tancredi, G., Rickman, H., \& Licandro, J. 1999, Astron. Astrophys. 352, 327

Fernández, J.A., Gallardo, T., \& Brunini, A. 2002, Icarus 159, 358

Fernández, Y.R. McFadden, L.A., Lisse, C.M., Helin, E.F., \& Chamberlin, A.B. 1997, Icarus 128,114

Fernández, Y.R., Jewitt, D.C., \& Sheppard, S.S. 2001, Astrophys. J. 553, L197

Fernández, Y.R., Abell, P.A., Pravec, P., French, L.M., Farnham, T.L., Gaffey, M.J., Hardersen, P.S., Kusnirak, P., Sarounova, L., \& Sheppard, S.S. 2003a, Bull. Amer. Astr. Soc. 35, [47.04]

Fernández, Y., Jewitt, D.C., \& Sheppard, S.S. 2003b, Astron. J. 123, 1050

Fernández, Y., Sheppard, S. \& Jewitt, D. 2003c, Astron. J. 126, 1563

Fernández, Y.R., Jewitt, D.C., \& Sheppard, S.S. 2005, Astron. J. 130, 308

Fleming, H.J. \& Hamilton, D.P. 2000, Icarus 148, 479

Fletcher, E., Fitzsimmons, A., Williams, I.P., Thomas, N., Ip, W.-H. 2000, in: A. Fitzsimmons, D. Jewitt \& R.M. West (eds.), Minor Bodies in the Outer Solar System, (Berlin: SpringerVerlag)

Fornasier, S., Barucci, M.A., \& Barbieri, C. 2004a, Astron. Astrophys. 422, L43

Fornasier, S., Dotto, E., Marzari, F., Barucci, M.A., Boehnhardt, H., Hainaut, O., \& de Bergh, C. $2004 \mathrm{~b}$, Icarus 172,221

Foster, M.J., Green, S.F., McBride, N., \& Davies, J.K. 1999, Icarus 141, 408

French, L.M. 2002, Bull. Amer. Astr. Soc. 33, [16.01], 368

Gladman, B., Kavelaars, J.J., Petit, J.-M., Morbidelli, A., Holman, M.J., \& Loredo, T. 2001, Astron. J. 122, 1051

Gomes, R. 2003, Icarus 161, 404

Green, D.W.E. 2005, IAU Circ. No. 8552

Greenberg, J.M., Mizutani, H., \& Yamamoto, T. 1995, Astron. Astrophys. 294, L35

Gronkowski, P. 2004, Astron. Nachr. 325, 343

Groussin, O., Lamy, P., \& Jorda, L. 2004, Astron. Astrophys. 413, 1163

Hainaut, O.R., Delahodde, C.E., Boehnhardt, H., Dotto, E., Barucci, M.A., Meech, K.J., Bauer, J.M., West, R.M., \& Doressoundiram, A. 2000, Astron. Astrophys. 356, 1076

Hicks, M.D., Buratti, B.J., Newburn, R.L., \& Rabinowitz, D.L. 2000, Icarus 143, 354

Hiroi, T., Kanno, A., Nakamura, R., Abe, M., Ishiguro, M., Hasegawa, S., Miyasaka, S., Sekiguchi, T., Terada, H., \& Igarishi, G. 2003, LPSCI 34, Abstr. 1425

Hiroi, T., Pieters, C.M., Rutherford, M.J., Zolensky, M.E., Sasaki, S., Ueada, Y., \& Miyamoto, 2004, LPSCI 35, Abstr. 1616

Hsieh, H.H., Jewitt, D.C., \& Fernández, Y.R. 2004, Astron. J. 127, 2997

Hsieh, H.H. 2004, Bull. Amer. Astr. Soc. 36, Abstr. [28.02] 
Hsieh, H.H. \& Jewitt, D. 2005, in: D. Lazzaro, S. Ferraz-Mello \& J.A. Fernández (eds.), Asteroids, Comes, Meteors, (Cambridge: Cambridge University Press)

Hsieh, H.H. \& Jewitt, D. 2005, Astrophys. J. 624, 1093

Jedicke, R. \& Metcalfe, K.J. 1998, Icarus 131, 245

Jewitt, D.C. \& Luu, J.X. 1990, Astron, J. 100, 933

Jewitt, D.C. 1992, in: A. Brahic, J.-C. Gerard \& J. Surdej (eds.) Proc. of the 30th Liége International Astrophysical Colloquium (Liége: Liége Press), 85

Jewitt, D. 1006, Earth, Moon, and Planets 72, 185\% From comets to asteroids: wheny hairy stars go bald

Jewitt, D. \& Kalas, P. 1998, Astrophys. J. 499, L103

Jewitt, D.C., Trujillo, Ch.A., \& Luu, J.X. 2000, Astron. J. 120, 1140

Jewitt, D.C. 2002, in: B. Warmbein (ed.), Proceedings of Asteroids, Comets, Meteors - ACM 2002, (Noordwijk, The Netherlands: ESA Publ. Division), ESA SP-500, 11

Jewitt, D.C. 2004, in: M.C. Festou, H.U. Keller \& H.A. Weaver (eds.), Comets II, (Tucson, Arizona: The University of Arizona Press), 659

Jewitt, D.C. \& Luu, J. 2004, Nature 432, 731

Jewitt, D.C., \& Sheppard, S.S., \& Porco, C. 2004, in: F. Bagenal, T.E. Dowling \& W.B. McKinnon (eds.), Jupiter, The planet, satellites and magnetosphere, 263

Jewitt, D. 2005, Astron. J. 129, 530

Jones, T., Lebofsky, L., Lewis, J., \& Marley, M. 1990, Icarus 88, 172

Kargel, J.S. 1991, Icarus 94. 368

Karlsson, O. 2004, Astron. Astrophys. 413, 1153

Kresák, L. 1979, in: T. Gehrels \& M.S. Matthews (eds.), Asteroids, (Tucson: University of Arizona Press), 289

Lamy, P.L., Toth, I., Fernández, Y.R., \& Weaver, H.A. 2004, in: M.C. Festou, H.U. Keller \& H.A. Weaver (eds.), Comets II, (Tucson, Arizona: The University of Arizona Press)

Lamy, P.L. \& Toth, I. 2005, Icarus, submitted

Larsen, J.A., Gleason, A.E., Danzl, N.M., Descour, A.S., McMillan, R.S., Gehrels, T., Jedicke, R., Monrani, J.L., \& Scotti, J.V. 2001, Astron. J. 121, 562

Laufer, D., Pat-El, I., \& Bar-Nun, A. 2005, Icarus, in press

Levison, H.F. 1996, in: T.W. Rettig \& J.M. Hahn (eds.), Completing the inventory of the solar system, (Astronomical Society of the Pacific Conference Proceedings), ASP Conf. Ser. 107, 173

Levison, H.F., Morbidelli, A., Dones, L., Jedicke, R., Wiegert, P.A., \& Bottke, W.F. 2002, Science 296, 2212

Licandro, J., Guerra, J.C., Campins, H., Di Martino, M., Lara, L.M., Gil-Hutton, R., \& Tozzi, G.P. 2002, Earth, Moon, and Planets 90, 495

Licandro, J., Campins, H., de Leon Cruz, J., Gil-Hutton, R., \& Lara-Lopez, L.M. 2003, Bull. Amer. Astr. Soc. 35, Abstr. [39.11]

Lien, D.J. 1998, Bull. Amer. Astr. Soc. 30, Abstr. [12.07], 1035

Lisse, C.M. 2002, Earth, Moon, and Planets 90, 497

Lisse, C.M., Fernández, Y.R., A’Hearn, M.F., Grün, E., Käufl, H.U., Osip, D.J., Lien, D.J., Kostiuk, T., Peschke, S.B., \& Walker, R.G. 2004, Icarus 171, 444

Lowry, S.C., Fitzsimmons, A., \& Collander-Brown, S. 2003, Astron. Astrophys. 397, 329

Lowry, S.C., \& Fitzsimmons, A. 2005, Mon. Not. R. Astr. Soc. 358, 641

Lowry, S.C., \& Weissman, P.R. 2003, Icarus 164, 492

Lunine, J. et al. 2004, in: M.C. Festou, H.U. Keller \& H.A. Weaver (eds.), Comets II, (Tucson, Arizona: The University of Arizona Press)

Luu, J.X. 1994, Publ. Astr. Soc. Pacific 106, 425

Luu, J., Jewitt, D., \& Cloutis, E. 1994, Icarus 109, 133

Luu, J.X., Jewitt, D.C., \& Trujillo, Ch. 2000, Astrophys. J. 531, L151

Luu, J.X. \& Jewitt, D.C. 2002, Annu. Rev. Astr. Astrophys. 40, 63

Mäkinen, J.T.T., Bertaux, J.-L., Pulkkinen, T.I., Schmidt, W., Kyrölä, H., Summanen, T., Quémarais, E., \& Lallement, R. 2001, Astron. Astrophys. 368, 292

Mann, I., Kimura, H., Biesecker, D.A., Tsurutani, B.T., Grün, E., McKibben, R.B., Liou, J.-C., MacQueen, R.M., Mukai, T., Guhathakurta, M., \& Lamy, P. 2004, Space Sci. Rev. 110, 269 
Marzari, F., Farinella, P., \& Vanzani, V. 1995, Astron. Astrophys. 299, 267

Marzari, F., Farinella, P., Davis, D.R., Scholl, H., \& Campo Bagatin, A. 1997, Icarus 126, 39

Marzari, F. \& Scholl, H. 1998a. Icarus 131, 41

Marzari, F. \& Scholl, H. 1998a, Astron. Astrophys. 339, 278

Marzari, F., Scholl, H., Murray, C., \& Lagerkvist, C. 2002, in: W.F. Bottke, A. Cellino, P. Paolicchi \& R.P. Binzel (eds.), Asteroids III, (Tucson, Arizona: The University of Arizona Press), 725

Marzari, F., Tricarico, P., \& Scholl, H. 2003, Icarus 162, 453

Meech, K.J., Hainaut, O.R., Boehnhardt, H., \& Delsanti, A. 2003, Bull. Amer. Astr. Soc. 35, Abstr. [39.12]

Meech, K.J., Hainaut, O.R., \& Marsden, B.G. 2004, Icarus 170, 463

Melita, M., Williams, I., \& Licandro, J. 2005, Asteroids, Comets, Meteors - ACM 2005, Abstract Book

Merline, W.J., Weidenschilling, S.J., Durda, D.D., Margot, J.L., \& Pravec, P., Storss, A.D. 2002, in: W.F. Bottke, A. Cellino, P. Paolicchi \& R.P. Binzel (eds.), Asteroids III, (Tucson, Arizona: The University of Arizona Press), 289

Michel, P., Benz, W., Tanga, P., \& Richardson, D.C. 2001, Science 294, 1696

Morbidelli, A., Levison, H.F., Tsiganis, K., \& Gomes, R. 2005, Nature 435, 462

Mousis, O. \& Alibert, Y. 2005, Mon. Not. R. Astr. Soc. 358, 188

Mueller, B.E.A., Hergenrother, C.W., Samarasinha, N.H., Campins, H., \& McCarthy, D.W. 2004, Icarus 171, 506

Mumma, M.J., Dello Russo, N., DiSanti, M.A., Magee-Sauer, K., Novak, R.E., Brittain, S., Rettig, T., McLean, I.S., Reuter, D.C., \& Xu, Li-H. 2001, Science 292, 1334

O'Brien, D.P. \& Greenberg, R. 2003, Icarus 164, 334

Noll, K.S., Weaver, H.A., \& Feldman, P.D 1996, in: K.S. Noll, H.A. Weaver \& P.D. Feldman (eds.), The Collision of Comet Shoemaker-Levy 9 and Jupiter. IAU Colloquium 156, (Cambridge, UK: Cambridge University Press)

Pan, M. \& Sari, R. 2005, Icarus 175, 343

Pittich, E.M., D'Abramo, G., \& Valsecchi, G.B. 2004, Astron. Astrophys. 422, 369

Press, W.H., Flannery, B.P., Teukolsky, S.A., \& Vetterling, W.T. 1986, in: Numerical Recipes - in Fortran The Art of Scientific Computing (Cambridge: Cambridge University Press)

Rabe, E. 1972, in: G.A. Chebotarev, E.I. Kazimirchak-Polonskaya \& B.G. Marsden (eds.), The Motion, Evolution of Orbits, and Origin of Comets, IAU Symposium No. 45, (Springer, New York), 55-60

Rodionov, A.V., Crifo, J.-F., Szegö, K., Lagerros, J., \& Fulle, M. 2002, Planet. Space Sci. 50, 983

Rousselot, P., Petit, J.-M., Poulet, F., Lacerda, P., \& Ortiz, J. 2003, Astron. Astrophys. 407, 1139

Saba, I., Capria, M.T., \& Cremonese, G. 2005, Mem. S. A. It. Suppl. Vol. 6, 137

Samarasinha, N.H. 2003, Adv. Space Res., in press.

Scheeres, D.J. 2005, LPSC 36, Abstr. No. 1919

Schulz, R. 2002, Astron. Astrophys. Rev. 11, 1

Sheppard, S.S., Jewitt, D.C., Trujillo, Ch.A., Brown, M.J.L., \& Ashley, M.C.B. 2000, Astron. J. 120,2687

Soderblom, L.A., Becker, T.L., Bennett, G., Boice, D.C.m. Britt, D.T., Brown, R.H., Buratti, B.J., Isbell, C., Giese, B., Hare, T., Hicks, M.D., Howington-Kraus, E., Kirk, R.L., Lee, M., Nelson, R.M., Oberst, J. Owen, T.C., Rayman, M.D., Sandel, B.R., Stern, S.A., Thomas, N., \& Yelle, R.V. 2002, Science 296, 1087

Stern, S.A. 1995, Astron. J. 110, 856

Stuart, J.S. 2001, Science 294, 1691

Tancredi, G. \& Sosa, A. 1996, Rev. Mex. Astr. Astrophys. 4, 118

Toth, I. 1999, Icarus 141, 420

Toth, I. 2000, Astron. Astrophys. 360, 375

Toth, I. 2001, Astron. Astrophys. 368, L25

Toth, I. 2005a, Astron. Astrophys., submitted.

Toth, I. 2005b, Astron. Astrophys., submitted. 
Toth, I., Lamy, P.L., \& Weaver, H.A. 2005, Icarus, in press.

Toth, I. \& Lisse, C.M. 2005, Icarus, submitted.

Trujillo, Ch.A., Jewitt, D.C., \& Luu, J.X. 2001, Astron. J. 122, 457

Vernazza, P., Monthé-Diniz, T., Birlan, M., Carvano, J.M., Strazzulla, G., Fulchignoni, M., \& Migliorini, A. 2005, Astron. Astrophys. 436, 1113

Weidenschilling, S.J. 2004, in: M.C. Festou, H.U. Keller \& H.A. Weaver (eds.), Comets II, (Tucson, Arizona: The University of Arizona Press)

Weaver, H.A., Sekanina, Z., Toth, I., Delahodde, C.E., Hainaut, O.R., Lamy, P.L., Bauer, J.M., A'Hearn, M.F., Arpigny, C., Combi, M.R., Davies, J.K., Feldman, P.D., Festou, M.C., Hook, R., Jorda, L., Keesey, M.S.W., Lisse, C.M., Marsden, B.G., Meech, K.J., Tozzi, G.P., \& West, R. 2001. Science 292, 1329

Weaver, H.A. 2004, Science 304, 1760

Weissman, P.R. 1986, Nature 320, 242

Weissman, P.R. 1989, in: S.K. Atreya, J.B. Pollack \& M.S. Matthews (eds.), Origin and Evolution of Planetary Satellite Atmospheres, (Tucson, Arizona: The University of Arizona Press), 230

Weissman, P.R., \& Levison, H.F. 1997, Astron. J. 488, L133

Weissman, P.R., Bottke, W.F. \& Levison, H.F. 2002 in: W.F. Bottke, A. Cellino, P. Paolicchi \& R.P. Binzel (eds.), Asteroids III, (Tucson, Arizona: The University of Arizona Press), 669

Weissman, P.R., Asphaug, E., \& Lowry, S.C. 2004, in: M.C. Festou, H.U. Keller \& H.A. Weaver (eds.), Comets II, (Tucson, Arizona: The University of Arizona Press)

Wilck, M. \& Mann, I. 1996, Planet. Space Sci. 44, 493

Wilck, \& Stern, S.A. 1999, Astron. Vestnik 33, 216

Yelle, R.V., Soderblom, L.A., \& Jokipii, J.R. 2004, Icarus 167, 30

Zahnle, K., Schenk, P., Sobieszczyk, S., Dones, L., \& Levison, H.F. 2001, Icarus 153, 111 